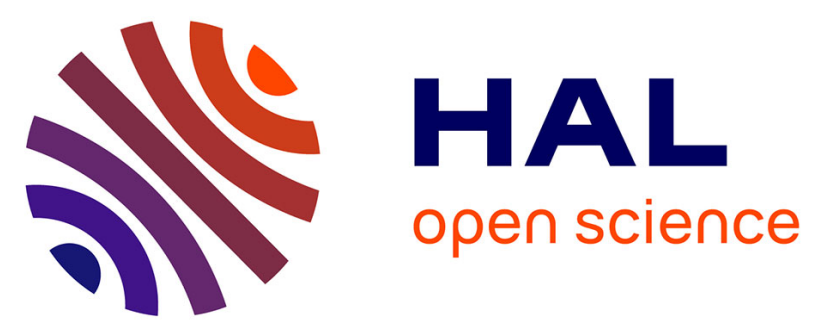

\title{
Efficient vectorized finite-difference method to solve the incompressible navier-stokes equations for 3-D mixed-convection flows in high-aspect-ratio channels
}

Abderrahmane Benzaoui, Xavier Nicolas, Shihe Xin

\section{- To cite this version:}

Abderrahmane Benzaoui, Xavier Nicolas, Shihe Xin. Efficient vectorized finite-difference method to solve the incompressible navier-stokes equations for 3-D mixed-convection flows in high-aspectratio channels. Numerical Heat Transfer, Part B Fundamentals, 2005, 48 (3), pp.277-302. 10.1080/10407790590959825. hal-00694583

\section{HAL Id: hal-00694583 \\ https://hal.science/hal-00694583}

Submitted on 4 May 2012

HAL is a multi-disciplinary open access archive for the deposit and dissemination of scientific research documents, whether they are published or not. The documents may come from teaching and research institutions in France or abroad, or from public or private research centers.
L'archive ouverte pluridisciplinaire HAL, est destinée au dépôt et à la diffusion de documents scientifiques de niveau recherche, publiés ou non, émanant des établissements d'enseignement et de recherche français ou étrangers, des laboratoires publics ou privés. 


\title{
Efficient vectorized finite difference method to solve the incompressible
}

\section{Navier-Stokes equations for 3D mixed convection flows in high aspect ratio}

\author{
channels
}

\author{
Abderrahmane BENZAOUI*, Xavier NICOLAS*, Shihe XIN ${ }^{\circ}$ \\ * LETEM, Université de Marne-La-Vallée, 77454 Marne-La-Vallée Cedex 2, France. \\ ○ LIMSI-CNRS, UPR 3251, BP 133, 91403 Orsay Cedex, France.
}

\begin{abstract}
Corresponding author :
Xavier NICOLAS

Mailing address for proof :

Université de Marne-La-Vallée, LETEM, Bât. Lavoisier, 77454 Marne-La-Vallée Cedex 2, France.
\end{abstract}

Abbreviated title for running head :

Vectorized finite differences for incompressible flows

\begin{abstract}
A very efficient vectorized code is tailored to solve 3D incompressible Navier-Stokes equations for mixed convection flows in high streamwise aspect ratio channels. It is based on Goda's algorithm, second order finite differences, incremental factorization method of ADI type, spectral decomposition of the 1D Laplace operators and TDMA algorithm. It is shown to be of second order both in space and time by a general method determining code convergence orders and to have good performance on NEC-SX5 super computer. It is validated through experiments of various Poiseuille-Rayleigh-Bénard flows with steady longitudinal, unsteady transverse and convectively unstable wavy rolls.
\end{abstract}




\section{Nomenclature}

$A, A_{e} \quad$ axial aspect ratios of the channel and of the unheated entrance zone, $L / H, L_{e} / H$

ADI Alternating Direction Implicit

$B \quad$ transversal aspect ratio of the channel, $l / H$

$c \quad$ dimensionless phase velocity of the transversal or wavy rolls, $\lambda f / U_{\text {mean }}$

$f \quad$ transversal or wavy roll frequency, $s^{-1}$

$f \quad$ variable replacing a set of variables verifying the same mathematical relation

$f_{\text {pert }} \quad$ frequency of the agitator sinusoidal excitation, $s^{-1}$

$g \quad$ gravitational acceleration, $\mathrm{m} / \mathrm{s}^{2}$

$H \quad$ channel height, $m$

$\vec{i}, \vec{k} \quad$ axial and upward vertical unit vectors

$l \quad$ channel width, $m$

$L, L_{e} \quad$ lengths of the channel and of the unheated entrance of the channel, $m$

$N \quad$ total number of mesh cells, $N_{x} \times N_{y} \times N_{z}$

$N_{t} \quad$ total number of time steps

$N_{x / y / z} \quad$ cell number in the $\mathrm{x}, \mathrm{y}$ or z directions

$p \quad$ dimensionless deviation of pressure from hydrostatic pressure

$\operatorname{Pr} \quad$ Prandtl number, $\nu / \alpha$

PRB Poiseuille-Rayleigh-Bénard

Ra Rayleigh number, $g \beta\left(T_{h}-T_{c}\right) H^{3} /(\nu \alpha)$

$R a_{/ /}^{*} \quad$ linear critical Rayleigh number of the PRB flow vis-a-vis the longitudinal rolls

$R a_{\perp}^{*} \quad$ same as $R a_{/ /}^{*}$ but for the transversal rolls

Re Reynolds number, $U_{\text {mean }} H / \nu$

$S_{f} \quad$ source term of the conservation equation for variable $\mathrm{f}$

$t \quad$ dimensionless time

$t_{C P U-t o t} \quad$ total CPU time for one simulation, $s$

$t_{C P U-u n i t} \quad$ CPU time per time step and per grid node, $s$

$T \quad$ température, $K$

$T_{c}, T_{h} \quad$ top cold wall and bottom hot wall temperatures of the channel, $K$

TDMA Tri-Diagonal Matrix Algorithm

$u, v, w \quad$ dimensionless streamwise, spanwise and vertical velocity components

$U, V, W \quad$ streamwise, spanwise and vertical velocity components, $\mathrm{m} / \mathrm{s}$ 
$U_{\text {mean }} \quad$ mean velocity of a flow in a rectangular channel, $\mathrm{m} / \mathrm{s}$

$U_{P o i s} \quad$ Poiseuille velocity profile in a rectangular channel (function of $\mathrm{x}$ and $\mathrm{y}$ ), $\mathrm{m} / \mathrm{s}$

$\vec{v}, \vec{V} \quad$ dimensionless and dimensional velocity vectors, $(u, v, w),(U, V, W)$

$v_{\text {max-pert }}$ dimensionless velocity excitation amplitude of the inlet agitator

$V_{r} \quad$ transversal roll phase velocity, $\lambda f, \mathrm{~m} / \mathrm{s}$

$W_{s} \quad$ saturation amplitude of the vertical velocity, $\mathrm{m} / \mathrm{s}$

$x, y, z \quad$ dimensionless axial, spanwise and vertical coordinates

$X, Y, Z \quad$ dimensional axial, spanwise and vertical coordinates, $m$

\section{Greek letters}

$\alpha \quad$ thermal diffusivity, $m^{2} / s$

$\beta \quad$ thermal expansion coefficient, $K^{-1}$

$\Delta t \quad$ dimensionless time step

$\Delta x, \Delta y, \Delta z$ dimensionless space step in $\mathrm{x}-, \mathrm{y}$ - and z-directions

$\epsilon_{f} \quad$ space or time relative error for variable $\mathrm{f}$

$\phi \quad$ increment of pressure, $p^{n+1}-p^{n}$

$\gamma_{\text {pert }} \quad$ agitator tilting angle, deg

$\lambda \quad$ transversal or wavy roll wave length, $m$

$\nu \quad$ kinematic viscosity, $\mathrm{m}^{2} / \mathrm{s}$

$\rho \quad$ mass per volume unit, $\mathrm{kg} / \mathrm{m}^{3}$

$\theta \quad$ reduced temperature, $\left(T-T_{c}\right) /\left(T_{h}-T_{c}\right)$

\section{Subscripts}

$\begin{array}{ll}\text { av } & \text { average } \\ \text { en } & \text { envelop or maximum amplitude } \\ i, j, k & \text { space indices in Cartesian space } \\ \text { pert } & \text { quantities relative to the inlet agitator } \\ \text { st, unst } & \text { steady and unsteady field } \\ \text { tot } & \text { total } \\ 0 & \text { analytical field } \\ / /, \perp, \approx & \text { relative to longitudinal, transversal or wavy rolls }\end{array}$

\section{Superscripts}

$n \quad$ time step number

* relative to the non-divergence-free velocity field or to a critical value 


\section{Introduction}

Poiseuille-Rayleigh-Bénard (PRB) flows are mixed convection flows in horizontal rectangular channels heated from below. They have been studied since the twenties, in the context of meteorology first and, since the sixties, to analyze theoretically [1-3] and experimentally [47] the stability of the numerous thermoconvective flow patterns that are liable to appear in these flows (see below). Since the eighties, PRB flows have enjoyed increasing attention of the fluid and thermal science community because of industrial applications, mainly in the processes of chemical vapor deposition (CVD) using cold wall rectangular thermal reactors to make depositions of thin solid films on substrates [8] and also in the context of the cooling of electronic equipments. A comprehensive and recent review on PRB flows can be found in [9].

In PRB flows, at relatively low Reynolds and Rayleigh numbers, the combination of RayleighBénard cells with a Poiseuille flow gives rise to very complex flow structures ranging from time-dependent transverse rolls to steady longitudinal rolls, time-dependent mixed rolls (superimposition of the two preceding ones) $[6,10,11]$, time-dependent oscillating rolls (wavy, snaking or varicose) $[1,12-15]$, etc. Time-dependent transversal rolls, with axis perpendicular to the Poiseuille flow direction, occur at very low Reynolds numbers. Since they are space and time periodic and quasi two-dimensional (2D) in the streamwise vertical planes, transversal rolls have been extensively studied by $2 \mathrm{D}$ numerical simulations ( $[2,16,17]$ among others) and more rarely by three-dimensional (3D) simulations [6,11,18-20]. Furthermore, as transversal roll flows formulate an open boundary problem with the transport of thermoconvective vortices out of the computational domain, they have been used as a test case to compare open boundary conditions. A benchmark was proposed by Sani and Gresho in 1989 [21] and several contributions have followed [22-24]. They have shown that Orlanski type open boundary conditions [25] are well suited to simulate the outlet conditions for $2 \mathrm{D}$ transversal roll flows.

3D numerical simulations of PRB flows are relatively recent and still not numerous because computations of such flows necessitate high aspect ratio channels and therefore large meshes and high computational costs. However the number of these simulations keeps growing because of the increasing capacities of the new generation computers. Before 1993, in the CVD context, 3D computations were limited to stationary problems or to parabolic simulations (the streamwise diffusions were neglected and solutions were computed by a marching technique in the flow direction). Therefore, only the steady transversal rolls at the entrance of the channels 
and the steady longitudinal rolls were able to be simulated [26-30]. Hosokawa et al. [31] and Chen and Lavine [20] have proposed unsteady and elliptic 3D simulations of PRB flows, but with periodic boundary conditions in the streamwise direction. The space development of the thermoconvective patterns has not been able to be taken into account.

To our knowledge, only Schröder and Bühler [19], Nobile and Onesti [12] and Lin et al. ( $[6,11,14,32]$ among others), with the Boussinesq model, and Evans and Greif $[18,33]$, Spall $[13,34]$ and Wang et al. [35], with non-Boussinesq models, have performed complete 3D numerical investigations of PRB flows, by taking into account the streamwise thermal and viscous diffusions and the space and time evolutions of the thermoconvective rolls. All these authors have simulated flows at $\operatorname{Pr} \approx 0.7$ (except [19] at $\operatorname{Pr}=530$ ), for moderate Reynolds numbers $R e \leq 50$ (except in [33] for $R e=100$ and 250) and for Rayleigh numbers $R a \leq 30000$ with Boussinesq models and till $R a=130700$ and a temperature ratio $r_{T}=\left(T_{h}-T_{c}\right) / T_{c}=2.33$ with non-Boussinesq models [13,33-35]. The discretization method is a finite difference or a finite volume method in all cases. The streamwise aspect ratio of the computational domain $A=L / H$ is smaller than 30 in all cases and the spanwise aspect ratio $B=l / H$ is between 2 and 12 , but generally less than 5 . In all papers, the total number of computational nodes is less than $N=2 \times 10^{5}$, except for a few computations, when the sensibility of the solution to the mesh size is tested: then $N$ reaches $3 \times 10^{5}$ or even $5 \times 10^{5}$ recently [35]. Two main methods are used to treat the velocity-pressure coupling: the iterative methods derived from the SIMPLE algorithm proposed by Patankar [36] (SIMPLER in [18,33,35] and SIMPLEC in [13,34]) and projection methods $[6,11,12,14,32]$. In some papers, the time integration is first or second order explicit $[6,11,14,19,32]$. In the others, it is implicit or semi-implicit with, generally, a standard iterative method to compute the solution: SOR, line by line or ADI methods.

In the present paper, we present a numerical procedure which computes the solutions of the 3D unsteady Boussinesq equations, discretized by a finite difference method on uniform or non-uniform structured grids, in high aspect ratio computational domains (say $100 \leq A \leq 250$, $B \approx 10$ ), with high spatial resolution (say $2 \times 10^{6} \leq N \leq 4 \times 10^{6}$, where $N$ is the total number of mesh cells), for reasonable time costs (less than 3 to 4 hours of CPU time on a vectorized supercomputer) and with second order space and time accuracies.

The development of a such procedure has been motivated by the fact that understanding, manipulating and controlling the thermoconvective patterns of PRB flows can become an im- 
portant research subject. In CVD rectangular reactors, for instance, operating conditions can result in steady longitudinal rolls which prevent from a uniform deposit [26-28]. To avoid the appearance of longitudinal rolls or, besides, other thermoconvective flows in these reactors, one solution is to strongly reduce the pressure below atmospheric pressure [8], which is an expensive operation. It has been shown that the transversal rolls, the longitudinal rolls and the wavy rolls are or can be convective instabilities depending on the flow parameters $[2,3,15]$, which means that they are very sensitive to external perturbations. An other solution to get more uniform depositions could be to control the steady longitudinal rolls and their secondary instabilities (the time periodic wavy and varicose rolls for instance) by introducing adequate perturbations (or excitations) in CVD reactors. However, the space growing rate of such thermoconvective rolls being relatively small, long streamwise aspect ratio channels, on the order of one or two hundred times the channel height, are necessary to get fully-developed patterns [15].

When we were seeking numerical methods able to solve the Boussinesq model on long computational domains and large grids for reasonable computational time cost, two main ideas oriented our choice. First, we have decided to only use direct algorithms, instead of iterative algorithms, to treat the velocity-pressure coupling and the time integration on the one hand and to compute the solutions of the different linear systems appearing in the procedure on the other hand. Second, in order to get high performances on vectorial super computers, we have decided to systematically use the highly vectorizable Thomas-Cholesky (or TDMA) algorithm in the different solvers. The different methods used are relatively classic but they are adapted and gathered here to provide an efficient code of second order space and time accuracies. The velocity-pressure coupling is handled by a two step projection method: the Goda's algorithm [37]. In a first "predictive" step, a second order time approximation of the velocity field is computed by solving a Helmholtz equation (the momentum equation) by an incremental factorization method of ADI type adapted from Hirsch (1989) [38]. In a second "projection" step, a Poisson equation is solved to get the increment of pressure by a direct matrix decomposition of the mono-dimensional (1D) Laplace operators into their eigen spaces (adapted from [39]). Then the approximate velocity field is explicitly corrected to ensure a strict mass conservation.

In the following, we first give the mathematical formulation of the treated problem: the Navier-Stokes equations under the Boussinesq approximation and the boundary conditions for 
the tested PRB configurations. We then detail the different steps of the numerical procedure. The validation of the numerical code is made in two steps. First, we verify that the space and time convergence orders of the computed solutions are actually of second order. A general method to determine the convergence orders of any Navier-Stokes solver is presented. We then validate the code through comparisons with experimental measurements, since, there is not any benchmark solution for 3D incompressible mixed convection flows in channels. The two first

experimental works, based on laser-Doppler anemometry measurements, are those of Chiu and Rosenberger (1987) [4] and Ouazzani et al. (1990) [5] : the former concerns steady longitudinal rolls in nitrogen $(P r=0.7)$ and the latter both unsteady transverse and steady longitudinal rolls in water $(\operatorname{Pr}=6.4)$. The third experimental work is the very recent work of Pabiou and his coauthors $[7,15,40,41]$, on $\mathrm{PRB}$ air flows $(\operatorname{Pr}=0.7)$, who, for the first time, have experimentally observed and analyzed the convectively unstable wavy rolls brought out by Clever and Busse (1991) [1] with a linear stability analysis. The aspect ratios of the experimental channel being $A=200$ and $B=10.38$, a large scale computation is necessary to reproduce this experiment. The global performances of the code are analyzed in the last section and the code efficiency is particularly enlightened through the last example.

\section{Mathematical equations and boundary conditions}

We are interested in computing PRB flows in horizontal channels of height $H$, width $W$ and length $L$, heated from below. As illustrated in Figure 1, the top horizontal wall is maintained at a uniform cold temperature $T_{c}$ and the bottom wall is heated at a higher temperature $T_{h}$ $\left(>T_{c}\right.$ ) either totally or partially. In the latter case there is an entrance length $L_{e}$ over which $T_{c}$ is imposed. The origin of the reference frame being placed at the beginning of the heated plate, the computational domain is defined by $(x, y, z) \in\left[-A_{e}, A-A_{e}\right] \times[0, B] \times[0,1]$ in nondimensional Cartesian coordinates, where $A_{e}=L_{e} / H$ is the streamwise entrance aspect ratio. The vertical lateral walls are adiabatic. A pressure-driven Poiseuille flow is imposed at the channel entrance and the incoming fluid can be either cold (at $\left.T_{c}\right)$ or hot $\left(\right.$ at $T_{h}$ ). Note that the velocity profile $U_{P o i s}(y, z)$ for " $3 \mathrm{D}$ " Poiseuille flow, i.e. in a $3 \mathrm{D}$ finite lateral extension channel, 
has been solved analytically in [42]. The dimensional velocity profile $U_{P o i s}(y, z)$ is given by:

$$
\frac{U_{\text {Pois }}(y, z)}{U^{\circ}}=6 z(1-z)+\frac{48}{\pi^{3}} \sum_{n=0}^{\infty} \frac{(-1)^{n+1} \cosh [(2 n+1) \pi(y-B / 2)] \cos [(2 n+1) \pi(z-1 / 2)]}{(2 n+1)^{3} \cosh [(2 n+1) \pi B / 2]}
$$

where $U^{\circ}=-\frac{H^{2}}{12 \mu} \frac{\partial P}{\partial X}$ is the dimensional average velocity of the " $2 \mathrm{D}$ " Poiseuille flow, i.e. in a $2 \mathrm{D}$ channel or between two infinite plates.

To simulate the PRB flows, we consider that the working fluid is Newtonian and incompressible, and that flows are governed by the 3D unsteady Navier-Stokes equations under the Boussinesq assumption. Giving the following reference quantities $H, U_{\text {mean }}, \rho U_{\text {mean }}^{2}$ and $H / U_{\text {mean }}$ for lengths, velocities, pressure and time, respectively, and defining the reduced temperature $\theta=\left(T-T_{c}\right) /\left(T_{h}-T_{c}\right)$, the non-dimensional governing equations are then:

$$
\left\{\begin{aligned}
\nabla \cdot \vec{v} & =0 \\
\frac{\partial \vec{v}}{\partial t}+(\vec{v} \cdot \nabla) \vec{v} & =-\nabla p+\frac{1}{R e} \nabla^{2} \vec{v}+\frac{R a}{\operatorname{Pr} R e^{2}} \theta \vec{k} \\
\frac{\partial \theta}{\partial t}+\vec{v} \cdot \nabla \theta & =\frac{1}{\operatorname{Pr} R e} \nabla^{2} \theta
\end{aligned}\right.
$$

where $\vec{v}=(u, v, w)$ is the velocity vector, $\nabla^{2}$ is the 3 D Laplace operator $\left(\frac{\partial^{2}}{\partial x^{2}}+\frac{\partial^{2}}{\partial y^{2}}+\frac{\partial^{2}}{\partial z^{2}}\right)$, $\vec{k}$ is the upward unit vector, $\operatorname{Pr}$ is Prandtl number $(=\nu / \alpha)$, Re is Reynolds number (= $\left.U_{\text {mean }} H / \nu\right)$, and $R a$ is Rayleigh number $\left(=g \beta\left(T_{h}-T_{c}\right) H^{3} /(\nu \alpha)\right)$. The boundary conditions for the dimensionless velocity and temperature fields are the following:

- at $z=0, \vec{v}=\overrightarrow{0}$; for $x \in\left[-A_{e}, 0\right], \theta=0$ or 1 and, for $x \in\left[0, A-A_{e}\right], \theta=1$;

- at $z=1, \vec{v}=\overrightarrow{0}$ and $\theta=0$;

- at $y=0$ and $B, \vec{v}=\overrightarrow{0}$ and $\partial \theta / \partial y=0$;

- at $x=-A_{e}, u=U_{\text {Pois }}(y, z) / U_{\text {mean }}, v=w=0$ and $\theta=0$ or 1 ;

- at $x=A-A_{e}$, an Orlanski type non-reflective boundary condition $[22,25]$ is used for $u$, $v, w$ and $\theta$. If $f$ represents $u, v, w$ and $\theta$, this condition writes: $\partial f / \partial t+\partial f / \partial x=0$. 


\section{Numerical methods}

\subsection{Time discretization}

Eqs. (2) are discretized in time by the second-order Adams-Bashforth scheme whose main characteristics are: a backward differencing for time derivatives $\left(\left(\frac{\partial f}{\partial t}\right)^{n+1}=\frac{3 f^{n+1}-4 f^{n}+f^{n-1}}{2 \Delta t}+\right.$ $\left.\mathcal{O}\left(\Delta t^{2}\right)\right)$, a fully implicit scheme for diffusion terms $\left(\left(\nabla^{2} f\right)^{n+1}\right)$ and an explicit treatment of nonlinear terms $\left((\vec{v} \cdot \nabla f)^{n+1}=2(\vec{v} \cdot \nabla f)^{n}-(\vec{v} \cdot \nabla f)^{n-1}+\mathcal{O}\left(\Delta t^{2}\right)\right)$, where $n$ is the time step number and $f$ represents $u, v, w$ and $\theta$. This time scheme yields:

$$
\begin{aligned}
\left(\frac{3}{2 \Delta t}-\frac{1}{\operatorname{Pr} R e} \nabla^{2}\right) \theta^{n+1} & =S_{\theta}^{n+1} \\
\left(\frac{3}{2 \Delta t}-\frac{1}{\operatorname{Re}} \nabla^{2}\right) \vec{v}^{n+1} & =\vec{S}_{\vec{v}}^{n+1}-\nabla p^{n+1}+\frac{R a}{\operatorname{Pr} \operatorname{Re}^{2}} \theta^{n+1} \vec{k} \\
\nabla \cdot \vec{v}^{n+1} & =0
\end{aligned}
$$

where $\vec{S}_{\vec{v}}=\left(S_{u}, S_{v}, S_{w}\right)$ and $S_{f}^{n+1}=\frac{4 f^{n}-f^{n-1}}{2 \Delta t}-2(\vec{v} \cdot \nabla f)^{n}+(\vec{v} \cdot \nabla f)^{n-1}$.

The boundary conditions do not require to be discretized in time, except the Orlanski condition at the channel outlet. In this boundary condition, the transport term is treated explicitly in time, in the same way as for the nonlinear convective terms because, an implicit treatment, combined with a second-order scheme in space, would have resulted in non-tridiagonal linear systems and would have not improved the stability of the time scheme.

\subsection{Incremental factorization method for solving Helmholtz equations}

Eqs. (3) are discretized in space on staggered grids by a second-order central scheme, for both the convective and diffusive terms. Uniform and non-uniform grids can be used but, only uniform grids are used here. Note that there is no stability problems linked with the use of this central scheme because, in the PRB flows considered here, the Reynolds or Péclet numbers are always small. The four first scalar equations of system (3) can be written in the form $\left(I-k \nabla^{2}\right) f^{n+1}=S_{f}$, where $I$ is the identity operator, $k$ is a constant and $S_{f}$ stands here for the whole source term. Therefore, they are 3D Helmholtz equations. As for a 1D Helmholtz equation, central differencing results in a tridiagonal linear system, the numerical method proposed here consists in factorizing the 3D Helmholtz operator into three 1D Helmholtz 
operators, in order to take advantage of Thomas-Cholesky algorithm (or TDMA algorithm). The first Helmholtz equation for temperature in (3) can be written:

$$
\left(I-\frac{2 \Delta t}{3 \operatorname{Pr} R e} \nabla^{2}\right) \theta^{n+1}=\frac{2 \Delta t}{3} S_{\theta}^{n+1}+\mathcal{O}\left(\Delta t^{3}\right)
$$

where $\left(I-\frac{2 \Delta t}{3 \operatorname{Pr} R e} \nabla^{2}\right)$ is the Helmholtz operator, which can be factorized into:

$$
\left(I-\frac{2 \Delta t}{3 \operatorname{Pr} R e} \nabla^{2}\right) \theta^{n+1}=\left(I-\frac{2 \Delta t}{3 \operatorname{Pr} R e} \frac{\partial^{2}}{\partial x^{2}}\right)\left(I-\frac{2 \Delta t}{3 \operatorname{Pr} R e} \frac{\partial^{2}}{\partial y^{2}}\right)\left(I-\frac{2 \Delta t}{3 \operatorname{Pr} R e} \frac{\partial^{2}}{\partial z^{2}}\right) \theta^{n+1}+\mathcal{O}\left(\Delta t^{2}\right)
$$

This factorization introduces an error of $\mathcal{O}\left(\Delta t^{2}\right)$ since the first neglected terms in Eq. (5) are of the form $\frac{4 \Delta t^{2}}{9 \operatorname{Pr}^{2} R e^{2}} \frac{\partial^{2}}{\partial x^{2}} \frac{\partial^{2}}{\partial y^{2}} \theta^{n+1}$. When this factorization is used in (4), it reduces the second-order scheme in time into a first-order scheme. In order to make use of Thomas-Cholesky algorithm and to keep a second-order accuracy at the same time, the idea of the incremental factorization method proposed here is to work with the increment of $\theta, \delta \theta=\theta^{n+1}-\theta^{n}$, and to factorize Helmholtz equation of $\delta \theta$ instead of $\theta$. Indeed, $\delta \theta=\theta^{n+1}-\theta^{n}$ is $\mathcal{O}(\Delta t)$ and the term $\frac{4 \Delta t^{2}}{9 \operatorname{Pr}^{2} R e^{2}} \frac{\partial^{2}}{\partial x^{2}} \frac{\partial^{2}}{\partial y^{2}} \delta \theta$ is $\mathcal{O}\left(\Delta t^{3}\right)$. The modified temperature equation reads then:

$$
\left(I-\frac{2 \Delta t}{3 \operatorname{Pr} R e} \nabla^{2}\right) \delta \theta=\left(\frac{2 \Delta t}{3 \operatorname{Pr} R e} \nabla^{2}-I\right) \theta^{n}+\frac{2 \Delta t}{3} S_{\theta}^{n+1}+\mathcal{O}\left(\Delta t^{3}\right)
$$

and can be put into the form:

$$
\begin{array}{r}
\left(I-\frac{2 \Delta t}{3 \operatorname{Pr} \operatorname{Re}} \frac{\partial^{2}}{\partial x^{2}}\right)\left(I-\frac{2 \Delta t}{3 \operatorname{Pr} \operatorname{Re}} \frac{\partial^{2}}{\partial y^{2}}\right)\left(I-\frac{2 \Delta t}{3 \operatorname{Pr} \operatorname{Re}} \frac{\partial^{2}}{\partial z^{2}}\right) \delta \theta= \\
\left(\frac{2 \Delta t}{3 \operatorname{Pr} R e} \nabla^{2}-I\right) \theta^{n}+\frac{2 \Delta t}{3} S_{\theta}^{n+1}+\mathcal{O}\left(\Delta t^{3}\right)
\end{array}
$$

without increasing the order of the time discretizing error. Equation (7) can be solved again by applying three successive Thomas-Cholesky algorithms.

\subsection{Goda's algorithm for velocity-pressure coupling}

In system (3), time integration and velocity and pressure coupling are computed by Goda's algorithm [37]. This algorithm is a projection method which consists in two main steps: prediction and projection. In the prediction step, after having obtained the temperature field, $\theta^{n+1}$, 
an approximated non-divergence-free velocity field, $\vec{v}^{*}$, is computed by solving the following momentum equation obtained by replacing $p^{n+1}$ by $p^{n}$ in second Eq. (3):

$$
\left(\frac{3}{2 \Delta t}-\frac{1}{R e} \nabla^{2}\right) \vec{v}^{*}=\vec{S}_{\vec{v}}^{n+1}-\nabla p^{n}+\frac{R a}{\operatorname{Pr} R e^{2}} \theta^{n+1} \vec{k}
$$

The boundary conditions for $\vec{v}^{*}$ are those of $\vec{v}^{n+1}:\left.\vec{v}^{*}\right|_{\partial \Omega}=\left.\vec{v}^{n+1}\right|_{\partial \Omega}$. As the components of Eq. (8) are Helmholtz equations, they are transformed into their incremental form and solved by using the incremental factorization method described in section 3.2 .

In the projection step, the non-divergence-free velocity field $\vec{v}^{*}$ is projected into a divergencefree subspace to get $\vec{v}^{n+1}$. This projection is done by solving:

$$
\left\{\begin{array}{l}
\frac{3\left(\vec{v}^{n+1}-\vec{v}^{*}\right)}{2 \Delta t}=-\nabla\left(p^{n+1}-p^{n}\right)=-\nabla \phi \\
\nabla \cdot \vec{v}^{n+1}=0 \\
\left.\vec{v}^{n+1} \cdot \vec{n}\right|_{\partial \Omega}=\left.\vec{v}^{*} \cdot \vec{n}\right|_{\partial \Omega}
\end{array}\right.
$$

where $\phi=p^{n+1}-p^{n}$ is the increment of pressure, $\vec{n}$ is the unit vector normal to the boundaries $\partial \Omega$ and the first equation is obtained by subtracting the Eq. (8) from the momentum equation in (3), considering that $\nabla^{2} \vec{v}^{*} \approx \nabla^{2} \vec{v}^{n+1}$. To get $\vec{v}^{n+1}$ from $\vec{v}^{*}$, we first compute $\phi$ by taking the divergence of the first equation of system (9) and homogeneous Neumann boundary conditions for $\phi$, i.e. by solving:

$$
\left\{\begin{array}{l}
\nabla^{2} \phi=\frac{3}{2 \Delta t} \nabla \cdot \vec{v}^{*} \\
\left.\frac{\partial \phi}{\partial \vec{n}}\right|_{\partial \Omega}=0
\end{array}\right.
$$

Once $\vec{v}^{*}$ and $\phi$ are known, $\vec{v}^{n+1}$ is explicitly computed from the first equation of (9) by: $\vec{v}^{n+1}=\vec{v}^{*}-\frac{2 \Delta t}{3} \nabla \phi$. The pressure field is updated by $p^{n+1}=p^{n}+\phi$.

\subsection{Partial diagonalization of the Laplace operator for solving Poisson equation}

After spatial discretization by finite differences, the Poisson Eq. (10), with homogeneous Neumann boundary conditions, is solved by a direct method based on reducing the 3D Laplace operator into $1 \mathrm{D}$ operators through the decomposition of discrete $\frac{\partial^{2}}{\partial y^{2}}$ and $\frac{\partial^{2}}{\partial z^{2}}$ into their eigenspaces. Thanks to these changes of bases, the matrix associated with the discrete Poisson equation becomes tridiagonal and can be inverted by Thomas-Cholesky's algorithm. 
Indeed, after having been discretized by a second order central difference scheme on a Cartesian grid of size $N_{x} \times N_{y} \times N_{z}$, where $N_{x}, N_{y}$ and $N_{z}$ are the total numbers of meshes in the directions $x, y$ and $z$ respectively, the Poisson Eq. (10), discretized on the node $(i, j, k)$ of coordinates $\left(x_{i}, y_{j}, z_{k}\right)$, can be written:

$$
\begin{aligned}
a_{i, i-1} \phi_{i-1, j, k}+a_{i, i} \phi_{i, j, k}+a_{i, i+1} \phi_{i+1, j, k} & +b_{j, j-1} \phi_{i, j-1, k}+b_{j, j} \phi_{i, j, k}+b_{j, j+1} \phi_{i, j+1, k}+ \\
c_{k, k-1} \phi_{i, j, k-1}+c_{k, k} \phi_{i, j, k}+c_{k, k+1} \phi_{i, j, k+1} & =S_{i, j, k}
\end{aligned}
$$

with

$$
S_{i, j, k}=\frac{3}{2 \Delta t}\left(\nabla \cdot \vec{v}^{*}\right)_{i, j, k}
$$

where $\phi_{i, j, k}=\phi\left(x_{i}, y_{j}, z_{k}\right), S_{i, j, k}=S\left(x_{i}, y_{j}, z_{k}\right)$ and where the coefficients $a_{p, q}, b_{p, q}$ and $c_{p, q}$ are the components of the three discrete operators $A, B$ and $C$ of second derivatives in the $x, y$ and $z$ directions respectively. $A, B$ and $C$ are tridiagonal matrices of sizes $N_{x} \times N_{x}, N_{y} \times N_{y}$ and $N_{z} \times N_{z}$ respectively. Using a tensorial notation and the Einstein contracted notation, Eq. (11) also writes:

$$
a_{i, l} \phi_{l, j, k}+b_{j, m} \phi_{i, m, k}+c_{k, n} \phi_{i, j, n}=S_{i, j, k}
$$

As the matrices $B$ and $C$ are diagonalizable, they can be written $B=P^{B} \Lambda^{B}\left(P^{B}\right)^{-1}$ and $C=P^{C} \Lambda^{C}\left(P^{C}\right)^{-1}$, where $\Lambda^{B}=\left(\lambda_{p, q}^{B}\right)_{1 \leq p, q \leq N_{y}}$ and $\Lambda^{C}=\left(\lambda_{p, q}^{C}\right)_{1 \leq p, q \leq N_{z}}$ are the diagonal matrices containing the eigenvalues of $B$ and $C$ and where $P^{B}=\left(p_{p, q}^{B}\right)_{1 \leq p, q \leq N_{y}}$ and $P^{C}=$ $\left(p_{p, q}^{C}\right)_{1 \leq p, q \leq N_{z}}$ are the associated base change matrices. Therefore, Eq. (13) may be written:

$$
a_{i, l} \phi_{l, j, k}+p_{j, p}^{B} \lambda_{p, p}^{B}\left(\left(p^{B}\right)^{-1}\right)_{p, m} \phi_{i, m, k}+p_{k, q}^{C} \lambda_{q, q}^{C}\left(\left(p^{C}\right)^{-1}\right)_{q, n} \phi_{i, j, n}=S_{i, j, k}
$$

As, for instance, $P^{B}\left(P^{B}\right)^{-1}=I d_{N_{y} \times N_{y}}$, which implies $p_{j, p}^{B}\left(\left(p^{B}\right)^{-1}\right)_{p, j}=\delta_{j, j}=1$ (where $\delta_{i, j}$ is the Kronecker symbol), by multiplying Eq. (14) by $\left(\left(p^{B}\right)^{-1}\right)_{p, j}\left(\left(p^{C}\right)^{-1}\right)_{q, k}$, we get:

$$
\begin{gathered}
a_{i, l}\left(\left(p^{B}\right)^{-1}\right)_{p, j}\left(\left(p^{C}\right)^{-1}\right)_{q, k} \phi_{l, j, k}+\lambda_{p, p}^{B}\left(\left(p^{B}\right)^{-1}\right)_{p, m}\left(\left(p^{C}\right)^{-1}\right)_{q, k} \phi_{i, m, k}+ \\
\lambda_{q, q}^{C}\left(\left(p^{B}\right)^{-1}\right)_{p, j}\left(\left(p^{C}\right)^{-1}\right)_{q, n} \phi_{i, j, n}=\left(\left(p^{B}\right)^{-1}\right)_{p, j}\left(\left(p^{C}\right)^{-1}\right)_{q, k} S_{i, j, k}
\end{gathered}
$$


which can also write:

$$
a_{i, l} \widetilde{\phi}_{l, p, q}+\lambda_{p, p}^{B} \widetilde{\phi}_{i, p, q}+\lambda_{q, q}^{C} \widetilde{\phi}_{i, p, q}=\left(a_{i, l}+\left(\lambda_{p, p}^{B}+\lambda_{q, q}^{C}\right) \delta_{i, l}\right) \widetilde{\phi}_{l, p, q}=\widetilde{S}_{i, p, q}
$$

where $\widetilde{\phi}_{l, p, q}=\left(\left(p^{B}\right)^{-1}\right)_{p, j}\left(\left(p^{C}\right)^{-1}\right)_{q, k} \phi_{l, j, k}$ and $\widetilde{S}_{i, p, q}=\left(\left(p^{B}\right)^{-1}\right)_{p, j}\left(\left(p^{C}\right)^{-1}\right)_{q, k} S_{i, j, k}$. Since the matrix $A+\left(\lambda_{p, p}^{B}+\lambda_{q, q}^{C}\right) I d_{N_{x} \times N_{x}}=\left(a_{i, l}+\left(\lambda_{p, p}^{B}+\lambda_{q, q}^{C}\right) \delta_{i, l}\right)_{1 \leq i, l \leq N_{x}}$ is tridiagonal, the computation of $\widetilde{\phi}_{l, p, q}$ knowing $\widetilde{S}_{i, p, q}$ necessitates to solve $N_{y} \times N_{z}$ tridiagonal linear systems of size $N_{x}$. The computation of the eigen values and eigen vectors of matrices $\Lambda^{B}, \Lambda^{C}, P^{B}$ and $P^{C}$ is made once in the preprocessing by using the vectorized Blas and Lapack routines.

To sum up, at each time step, Eqs. (3) are solved in three steps : in the first one, we calculate $S_{\theta}^{n+1}$ and the right-hand-side of the factorized Helmholtz Eq. (7) for $\delta \theta$. This equation is solved by the incremental factorization method and $\theta^{n+1}$ is recovered. In the second step, to get the approximated non-divergence-free velocity field $\vec{v}^{*}$, we compute $S_{u}^{n+1}, S_{v}^{n+1}, S_{w}^{n+1}$ and solve momentum Eq. (8), by using incremental factorization method once again. In the third and final step, $\nabla \cdot \vec{v}^{*}$ is calculated, the Poisson Eq. (10) for $\phi$ is solved by the just described method and $\vec{v}^{n+1}$ and $p^{n+1}$ are explicitely updated.

\section{Validation of the numerical procedure}

\subsection{Determination of the space and time convergence orders}

As mentioned before, second order finite difference schemes in space and in time are used in the discretization of the model equations. The boundary conditions are also discretized using second order schemes. This signifies that the consistency orders of the discretized equations are of second order. Here, we are going to show that the convergence orders of the computed solutions, at the end of the whole numerical procedure, are effectively of second order. Generally, the numerical solution should be compared to an exact analytical solution of a simplified problem in which some terms of the Navier-Stokes equations are dropped. Here, the method used to determine the convergence orders is more general, in the sense that it can be easily adapted to any type of partial differential equations. It consists in introducing an appropriate forcing term in the equation whose convergence order has to be determined, so that the solution computed with this forcing term converges to a given analytical field $\left(\overrightarrow{v_{0}}, p_{0}, \theta_{0}\right)$. The convergence order is obtained by comparing the computed solution and the analytical field. 
More precisely, in the case of the equations of the present paper, we first construct an analytical field $\left(\overrightarrow{v_{0}}, p_{0}, \theta_{0}\right)$ satisfying the mass conservation equation and a set of given boundary conditions in a given computational domain. Here, the convergence orders are calculated in a differentially-heated cubic computational domain $(\mathrm{A}=\mathrm{B}=1)$, with uniform meshes in all space directions $(\Delta x=\Delta y=\Delta z)$, with no-slip boundary conditions and conducting walls:

$$
\left\{\begin{array}{l}
\vec{v}(0, y, z)=\vec{v}(1, y, z)=\vec{v}(x, 0, z)=\vec{v}(x, 1, z)=\vec{v}(x, y, 0)=\vec{v}(x, y, 1)=\overrightarrow{0} \\
\theta(x, y, 0)=1 ; \theta(x, y, 1)=0 ; \theta(0, y, z)=\theta(1, y, z)=\theta(x, 0, z)=\theta(x, 1, z)=1-z \\
\frac{\partial p}{\partial x}(0, y, z)=\frac{\partial p}{\partial x}(1, y, z)=\frac{\partial p}{\partial y}(x, 0, z)=\frac{\partial p}{\partial y}(x, 1, z)=\frac{\partial p}{\partial z}(x, y, 0)=\frac{\partial p}{\partial z}(x, y, 1)=0
\end{array}\right.
$$

Note that the boundary conditions for pressure have been chosen in accordance with the boundary conditions of Poisson Eq. (10) in the velocity-pressure decoupling algorithm. Then, using the same methods and algorithms as those of section 3, we compute the solution of Eqs. (2) in which appropriate forcing terms $\overrightarrow{F_{v_{0}}}$ and $F_{\theta_{0}}$ are added, i.e. the following equations are solved:

$$
\left\{\begin{aligned}
\nabla \cdot \vec{v} & =0 \\
\frac{\partial \vec{v}}{\partial t}+(\vec{v} \cdot \nabla) \vec{v}+\nabla p-\frac{1}{R e} \nabla^{2} \vec{v}-\frac{R a}{\operatorname{Pr} R e^{2}} \theta \vec{k} & =\overrightarrow{F_{v_{0}}} \\
\frac{\partial \theta}{\partial t}+\vec{v} \cdot \nabla \theta-\frac{1}{\operatorname{Pr} R e} \nabla^{2} \theta & =F_{\theta_{0}}
\end{aligned}\right.
$$

The forcing terms are constructed such that the solution converges to the chosen analytical fields, i.e. the forcing terms are equal to the left-hand-side of Eqs. (18) for $\left(\overrightarrow{v_{0}}, p_{0}, \theta_{0}\right)$ :

$$
\left\{\begin{array}{l}
\overrightarrow{F_{v_{0}}}=\frac{\partial \overrightarrow{v_{0}}}{\partial t}+\left(\overrightarrow{v_{0}} \cdot \nabla\right) \overrightarrow{v_{0}}+\nabla p_{0}-\frac{1}{R e} \nabla^{2} \overrightarrow{v_{0}}-\frac{R a}{\operatorname{Pr} e^{2}} \theta_{0} \vec{k} \\
F_{\theta_{0}}=\frac{\partial \theta_{0}}{\partial t}+\overrightarrow{v_{0}} \cdot \nabla \theta_{0}-\frac{1}{\operatorname{Pr} R e} \nabla^{2} \theta_{0}
\end{array}\right.
$$

To determine the error only due to the space discretization, steady analytical fields $\left(\overrightarrow{v_{0-s t}}(x, y, z)\right.$, $\left.p_{0-s t}(x, y, z), \theta_{0-s t}(x, y, z)\right)$ are chosen so as to the time error is vanished. Here, the following 
steady fields, satisfying the boundary conditions (17) and the continuity equation, are used:

$$
\left\{\begin{array}{l}
u_{0-s t}=2(1-\cos (2 \pi x)) \sin (2 \pi y) \sin (2 \pi z) \\
v_{0-s t}=\sin (2 \pi x)(\cos (2 \pi y)-1) \sin (2 \pi z) \\
w_{0-s t}=\sin (2 \pi x) \sin (2 \pi y)(\cos (2 \pi z)-1) \\
\theta_{0-s t}=1-z+x^{2}(1-x)^{2} y^{2}(1-y)^{2} z(1-z) \\
p_{0-s t}=\sin \left(\pi x+\frac{\pi}{2}\right) \sin \left(\pi y+\frac{\pi}{2}\right) \sin \left(\pi z+\frac{\pi}{2}\right)
\end{array}\right.
$$

Then, we can define the relative space errors $\epsilon_{f}$ between the computed fields $f$ (where $f=u, v, w, \theta$ or $p$ ) and the analytical fields $f_{0}$ :

$$
\epsilon_{f}=\frac{\max _{(i, j, k) \in\left[1 ; N_{x}\right] \times\left[1 ; N_{y}\right] \times\left[1 ; N_{z}\right]}\left|f(i, j, k)-f_{0}(i, j, k)\right|}{\max _{(i, j, k) \in\left[1 ; N_{x}\right] \times\left[1 ; N_{y}\right] \times\left[1 ; N_{z}\right]}\left|f_{0}(i, j, k)\right|}
$$

These errors are drawn on Figure 2(a) as function of the space step $\Delta x$ (the meshes correspond to $N_{x} \times N_{y} \times N_{z}=20^{3}, 30^{3}, 40^{3}, 60^{3}$ and $80^{3}$ ), after a sufficiently long integration time to be able to consider that the flow is steady. As a maximum discrepancy of only $1 \%$ exists between the values of $\epsilon_{u}, \epsilon_{v}$ and $\epsilon_{w}$, an average value $\epsilon_{u, v, w}$ is drawn on Figure 2(a). The slopes of the curves $\log \left(\epsilon_{f}\right)=f(\log (\Delta x))$ being equal to 2 , the space convergence order of the computed fields is then equal to the space consistency order of the discretized equations: $\epsilon_{f} \sim \mathcal{O}\left(\Delta x^{2}\right)$.

To determine the time convergence order, the way of proceeding is the same as for the space convergence order. The same computational domain is kept, but the boundary conditions and the analytical fields become unsteady. They are defined by:

$$
\left\{\begin{array}{l}
B C_{\text {unst }}=B C_{s t} \times h(t) \\
f_{0-\text { unst }}=f_{0-s t} \times h(t)
\end{array}\right.
$$

where $B C_{\text {unst }}$ and $B C_{s t}$ means "unsteady" and "steady boundary conditions", where $B C_{s t}$ represents all the boundary conditions given by Eqs. (17), where $f$ stands for $u, v, w, \theta$ and $p$ and the time function $h(t)$ equals $\exp \left(\frac{t}{2}\right)$ or $\sin \left(\frac{\pi t}{2}\right)$.

Using a uniform mesh with $N_{x} \times N_{y} \times N_{z}=30^{3}$ and starting from the initial condition given by Eqs. (22) at time $t=0$, the errors $\epsilon_{f}$ computed at the fixed time $t=4$ are drawn as a function of $\Delta t$, for the two functions $h(t)$, on Figure 2(b). Note that, to only get the time error, the space differential operators of the source terms (19) are computed using their finite difference 
discrete form (the same as the one used to discretize the left-hand-side of Eqs. (18)) instead of their continuous form which is used for the determination of the space convergence order. This permits to make the space error vanished so that only the time error is evaluated. The computation of the errors $\epsilon_{u}, \epsilon_{v}$ and $\epsilon_{w}$ is of course made using the final velocity field, $\vec{v}^{n+1}$, and not the intermediate one, $\vec{v}^{*}$. Figure $2(\mathrm{~b})$ permits to conclude that the time convergence order of the present code is of second order like the space convergence order: $\epsilon_{f} \sim \mathcal{O}\left(\Delta x^{2}, \Delta t^{2}\right)$.

\subsection{Comparisons with experimental results}

In order to validate our numerical code, in a first step, we have carried out quantitative comparisons with the experiments of Chiu and Rosenberger (1987) [4] and Ouazzani et al. (1990) [5]. The comparisons only concern the velocity components since these experiments are only based on LDA measurements of velocity profiles and signals. In a second step, we have performed a large scale computation to reproduce one of the experiments of Pabiou (2003) [7] (see also [41]) about the measurement of the frequency, the wave length and the phase velocity of the wavy rolls obtained by introducing a sinusoidal excitation, at the entrance of the channel, in a longitudinal roll flow.

\subsubsection{Test case 1: Chiu and Rosenberger's experiments}

The first test case, extracted from Chiu and Rosenberger's experiments [4], concerns the space development of steady longitudinal rolls from the beginning of the heated zone, in a nitrogen PRB flow. The dimensions of the heated zone are $\left(L-L_{e}\right) \times W \times H=87.5 \mathrm{~cm} \times$ $15.24 \mathrm{~cm} \times 1.58 \mathrm{~cm}$. This zone is preceded by an isothermal zone at $T_{c}$ of same section and of length $L_{e}=67.5 \mathrm{~cm}$. For this test case, the flow enters the channel at $T_{c}$. Non-dimensional flow parameters, calculated at the average temperature $\left(T_{h}+T_{c}\right) / 2$, are $R e=42, R a=4878$ and $\operatorname{Pr}=0.71$. Spanwise profiles of the longitudinal velocity component $U$ are measured by a commercial LDA system at $Z=0.2 H$ and $Z=0.5 H$, for several $X$ positions. These profiles are presented on Figures 3. They are compared with our numerical results and those of Nobile and Onesti (1996) [12]. Nobile and Onesti (1996) [12] used a computational domain of size $A \times B=30 \times 9.65$ with a mesh of size $N_{x} \times N_{y} \times N_{z}=126 \times 62 \times 30$. Our results are calculated in a computational domain whose dimensions are $A=40, A_{e}=10$ and $B=9.65$ with a uniform mesh of size $N_{x} \times N_{y} \times N_{z}=480 \times 120 \times 32$. The time step is $\Delta t=0.005$ 
and the total integration time is 50 . The boundary conditions are the same as those given in section 2 , with $\theta=0$ at $x=-A_{e}$.

Figure 3 shows that our results situate between the experimental profiles and the numerical profiles of Nobile and Onesti (1996) [12]. Calculations agree well with the experiments, particularly at $Z=0.5 H$ (see the three graphs at the top of Figure 3). At $Z=0.2 H$ (see the three graphs at the bottom of Figure 3), a larger discrepancy between experiments and calculations appears at $X=10$ and $20 \mathrm{~mm}$. This discrepancy might be attributed to conjugate heat transfer effects not taken into account in the numerical studies, but it might also be due to a slight uncertainty in the positioning of the laser in the experiments since, at $Z=0.2 H$, the $U$-velocity vertical gradient is maximum. Indeed, we have verified that the experimental profiles at $X=10$ and $20 \mathrm{~mm}$ are numerically recovered by plotting $U$-velocity at $Z=0.22 \mathrm{H}$, instead of $Z=0.2 H$. The difference of $0.02 H=0.316 \mathrm{~mm}$ would represent a positioning error, given the fact that the thickness of a laser sheet is approximately of $0.3 \mathrm{~mm}$.

\subsubsection{Test case 2: Ouazzani et al.'s experiments}

The second test case, extracted from Ouazzani et al.'s experiments [5], concerns fullyestablished steady longitudinal rolls and fully-established unsteady periodic transversal rolls, in water PRB flows $(\operatorname{Pr}=6.4)$. The channel height $H$, width $W$ and length $L$ are respectively equal to $4.15 \pm 0.05 \mathrm{~mm}, 15.05 \pm 0.05 \mathrm{~mm}$ and $\sim 115 \mathrm{~mm}$. The bottom wall is totally heated at temperature $T_{h}$ and the incoming water is also heated at $T_{h}$. Space profiles and temporal signals of vertical velocity are measured through a LDA system. The boundary conditions are the same as those given in section 2 , with $A_{e}=0$ and with $\theta=1$ at $x=-A_{e}$. Note that, in spite of the likely existence of small conjugate heat transfers in the experiments due to the use of glass walls and water flows, the vertical lateral walls are considered as adiabatic in the numerical computations. Indeed, we have verified that adiabatic side walls are much more appropriate to reproduce the results of Ouazzani et al.'s experiments [5] than conductive side walls $(\theta=1-z$ at $y=0$ and $B)$.

On Figure 4, we compare the computed and experimentally measured spanwise profiles of the vertical velocity component $W$, at $x=15.7$ and $z=0.5$, in a flow made of four longitudinal rolls, at $\operatorname{Re}=1.02, \operatorname{Pr}=6.4$ and at different Rayleigh numbers. A computational domain of size $A \times B=40 \times 3.63$, with a mesh of size $N_{x} \times N_{y} \times N_{z}=480 \times 90 \times 32$, and a 
time step $\Delta t=0.005$ with a total integration time of 30 are used for these simulations. The amplitude of the $W$ profiles increases with Rayleigh number. The agreement between the experimental and computed results is very good at the two higher Rayleigh numbers $R a=3494$ and 4724. It is slightly less good at $R a=2429$. This may be due to the uncertainty in the experimental measurement of the Rayleigh number because it induces a non negligible error on the experimental measurement of $W$. Indeed, following the analysis of Dubois and Bergé (1978) [43] on the vertical velocity amplitude in the case of Rayleigh-Bénard flows, the amplitude of $W$ in a longitudinal roll flow can be considered as being proportional to $\left(\frac{R a-R a_{/ /}^{*}}{R a_{/ /}^{*}}\right)^{1 / 2}$ at moderate Rayleigh and Reynolds numbers (where $R a_{/ /}^{*}$ is the critical Rayleigh number for the appearance of the longitudinal rolls determined by linear stability analysis). Therefore, only taking into account the experimental uncertainties on $H$ and $\Delta T$ and knowing that $R a_{/ /}^{*}=1849$ [9], it can be shown that the discrepancy between the experimental and theoretical values of $W$ is approximately $9 \%$ at $R a=2429,4 \%$ at $R a=3494$ and $3 \%$ at $R a=4724$.

The following comparisons concern transversal roll flows. The simulations are performed for a computational domain of size $A \times B=27 \times 3.63$ with a mesh of size $N_{x} \times N_{y} \times N_{z}=400 \times 60 \times 30$. The dimensionless time step is $\Delta t=2 \times 10^{-3}$ and the total integration time is 40 . Once a fully-established transversal roll flow is obtained, the extrema $W_{\max }(X)$ and $W_{\min }(X)$ of the time signals $W(X, t)$ are computed at each axial position $X$, at $(Y, Z)=(l / 2, H / 2)$. The amplitude (or envelop) of $W$, defined by $W_{e n}(X)=\frac{W_{\max }(X)-W_{\min }(X)}{2}$, and its average value $W_{a v}(X)=\frac{W_{\max }(X)+W_{\min }(X)}{2}$ can then be calculated. On Figure 5 , we present $W(X)$ at a fixed time, $W_{e n}(X)$ and $W_{a v}(X)$, at $R a=4700$ and $R e=0.21$. Three zones appear in the channel: (1) an inlet zone where the average vertical velocity $W_{a v}$ is not equal to zero; (2) a central zone with a fully-established space and time periodic transversal roll flow characterized by a zero average vertical velocity $W_{a v}$ and a constant value of the vertical velocity envelop $W_{e n} ;(3)$ an outlet zone, with the envelop $W_{e n}$ slightly perturbed by the Orlanski outlet boundary conditions. In the following, the constant envelop $W_{e n}$ in zone (2) is called the saturation vertical velocity and is noted $W_{s}$. On Figure 6, we compare the experimentally measured and computed values of $W_{s}^{2}$ at $R e=0.21$, for different $R a$. The experimental error bars on the determination of $R a$ are also given by taking into account the uncertainties on the measure of $H$ and $\Delta T$. Once again, a good agreement is observed between the experimental and numerical values of $W_{s}$. The 
numerical results of Figure 6 can be correlated with a precision of $3 \%$, for $1800 \leq R a \leq 4700$, by:

$$
W_{s}=455.87\left(\frac{R a-1725}{1725}\right)^{0.47} \quad(\mu m / s)
$$

Following the weakly non linear theory of Müller et al. [2], the value 1725 in the above correlation should correspond to the linear critical Rayleigh number $R a_{\perp}^{*}$ for the appearance of the transversal rolls. At $R e=0$ and $B=3.63$, the linear stability theory gives $R a_{\perp}^{*}=1730$ [42].

In zone (2) of Figure 5, the curve $W=f(X)$ permits to determine the wave length $\lambda$ of the transversal rolls, while a signal $W=f(t)$ at point $X=65 \mathrm{~mm} \approx L / 2,(Y, Z)=(l / 2, H / 2)$ permits to determine the frequency $f$ of the transversal rolls. The phase velocity $V_{r}$ of the transversal rolls is then equal to the product $\lambda f$. It is well known that the dimensionless phase velocity $c_{\perp}=V r / U_{\text {mean }}$ linearly decreases with $R a$ and is independent of $R e$ (for a review on this subject, see [9]). On Figure 7, we compare the experimental curve $c_{\perp}=f(R a)$ obtained by Ouazzani et al. (1990) [5] to those obtained by 2D numerical simulations by Nicolas et al. (1997) [17] and by the present 3D numerical simulations. In the case of the 3D computations, the error bars on $c_{\perp}$, which are principally due to an uncertainty of $3 \%$ on the determination of $\lambda$ in zone (2) (see Figure 5), are drawn on Figure 7. Only the 3D simulations recover the strong decrease of $c_{\perp}$ with respect to $R a$ observed in the experiments, proving that this behavior is due to the lateral confinement. It can also be noted that the $2 \mathrm{D}$ and $3 \mathrm{D}$ numerical results perfectly agree with the linear stability theory [9] since, at $\operatorname{Pr}=6.4$, it gives a critical phase velocity $c_{\perp}{ }^{*}$ equal to 1.28 when $B \rightarrow \infty$ and equal to 1.5 at $B=3.6$.

\subsubsection{Test case 3: Pabiou et al.'s experiments}

Using a temporal linear stability analysis, Clever and Busse (1991) [1] have theoretically shown that the longitudinal rolls are unstable vis-a-vis oscillating or wavy rolls, in PRB flows between two infinite plates. In the case of air flows $(\operatorname{Pr}=0.7)$, for instance, they show that the longitudinal rolls become linearly unstable vis-a-vis the wavy rolls, approximately for $R e \geq 80$ and for $R a \geq 2500$. To observe these rolls, Pabiou et al. (2003) [7,15] set up a PRB experiment in an air channel of size $L \times W \times H=2800 \times 150 \times 14.45 \mathrm{~mm}^{3}$ for the following parameters: $\operatorname{Pr}=0.7, R a>3000$ and $R e>120$. Unfortunately the wavy rolls were not observed: in fact, in a rectangular channel of finite longitudinal and transversal extensions, Nicolas et al. (2003) [40] 
and Pabiou et al. (2003) [7,15] have numerically and experimentally shown that the wavy rolls are a convective instability of the longitudinal rolls. This have permitted to explain why the wavy rolls had never been experimentally and numerically observed before. Indeed, to make the wavy rolls appear in a PRB flow, it is necessary to continuously excite the longitudinal rolls: a white noise is added to the inlet Poiseuille profile in the numerical simulations of Nicolas et al. (2003) [40] and Pabiou et al. (2003) [15], while an agitator oscillates in the channel core, upstream the bottom heated plate, in the experiments of Pabiou et al. (2003) [7,15,41].

We propose here to numerically reproduce one of these experiments for $R a=6300, R e=$ 162, $A=250, A_{e}=2, B=10.38$ and adiabatic vertical lateral walls. The mesh size used is $N_{x} \times N_{y} \times N_{z}=1250 \times 120 \times 30$ and the time step is $\Delta t=0.02$. We performed numerical simulations of the space and time development of wavy roll flows by introducing, at the channel inlet, excitations describing the agitator action. As the mathematical translating of this action is hard to precisely write, a rough approximation permits to consider that the agitator creates a sinusoidal perturbation of the vertical and streamwise velocity components of the form $[7,15,41]$ :

$$
\overrightarrow{v_{\text {pert }}}\left(x_{\text {pert }}, y, z_{\text {pert }}, t\right)=v_{\text {max }- \text { pert }} \sin \left(2 \pi \frac{H}{U_{\text {mean }}} f_{\text {pert }} t\right)\left(\sin \gamma_{\text {pert }} \vec{i}+\cos \gamma_{\text {pert }} \vec{k}\right)
$$

where $x_{\text {pert }}$ and $z_{\text {pert }}$ correspond to the agitator dimensionless location, $v_{\text {max-pert }}$ is the dimensionless amplitude of the velocity excitation, $f_{\text {pert }}$ is the forcing frequency in Hertz and $\gamma_{\text {pert }}$ is the tilting angle of the arms that support the stirring rod and that are connected to a driving motor. Note that Eq. (24) does not take into account the influence of the movement of these arms that are placed in the boundary layer of the vertical wall. For the above flow parameters, Pabiou and Mergui $[7,41]$ find that the inlet excitation frequency for which the space growth rate of the wavy instabilities is maximum is $f_{\text {pert }}=3 \mathrm{~Hz}$. More precisely, the agitator characteristics corresponding to this excitation are $[7,44]: x_{p e r t}=-2, z_{\text {pert }}=0.286$, $v_{\text {max }- \text { pert }}=0.37, f_{\text {pert }}=3 \mathrm{~Hz}$ and $\gamma_{\text {pert }}=46 \mathrm{deg}$. To try to reproduce this agitator numerically, the time dependent velocity vector given by Eq. (24) is introduced in the Poiseuille profile of the inlet boundary condition placed at $x=-A_{e}=-2$ (see section 2): this Poiseuille profile is kept, except along the two horizontal mesh lines in the neighborhood of $z=z_{\text {pert }}$ where the velocity vectors are given by Eq. (24). Furthermore, a flow rate correction is ensured at each time step, in order to keep the average velocity $u_{\text {mean }}$ at inlet. 
In Figure 8, we present two numerical results obtained with two different amplitudes of excitations at the channel inlet. The excitation of Figure 8(a) corresponds (in theory) to the experimental one $\left(v_{\max -p e r t}=0.37\right)$ and the excitation of Figure $8(\mathrm{~b})$ is for a ten times higher dimensionless amplitude: $v_{\text {max-pert }}=3.7$. In both simulations, at time $t=0$ (not shown in Figure $8(\mathrm{~b})$ ), the initial condition is the same fully established ten longitudinal roll flow. This steady longitudinal roll flow has been obtained for the flow parameters given above, without introducing any excitation in the channel, starting from a Poiseuille flow at the cold temperature $\theta=0$ and by integrating during 350 dimensionless time units, with the bottom plate uniformly heated at temperature $\theta=1$ (refer to [15] to see the space and time development of these steady longitudinal rolls). In Figure 8 , for $t>0$ and during all the simulation, the excitation described above is imposed at channel inlet. The resulting disturbances propagate downstream in growing. Wavy rolls can be observed from time $t=160$ in Figure 8 (a) for the excitation of smallest amplitude and, as soon as time $t=80$, in Figure 8(b), for the excitation of higher amplitude. In both cases, the wavy roll flows can be considered as fully-developed for $t>250$. These fully-developed wavy roll flows are shown at time $t=400$ in Figures $8(\mathrm{a})$ and $8(\mathrm{~b})$.

Two main differences appear between these two cases. The first one concerns the wavy roll growth length: as it could have been expected because of the factor ten between the two excitation amplitudes, this growth length is much longer in the first case (around 200H) than in the second one (around 50H). Note that, like in the simulation of Figure 8(a), in the corresponding experiment of Pabiou and Mergui [7,44], the wavy rolls also appear close to the channel end and are not completely fully-developed. The second difference between the two simulations of Figure 8 concerns the number of wavy rolls. In the first case, as expected, ten wavy rolls develop, while, in the second case, the amplitude of the velocity excitation at inlet (equal to 3.7 times the average flow velocity) breaks the ten longitudinal rolls of the basic flow and, in an unexpected way, twelve wavy rolls develop. Finally, one can also note that there is no roll oscillation on the channel axes of Figures $8(\mathrm{a})$ and $8(\mathrm{~b})$. This behavior is due to the axial symmetry of all the boundary conditions (even the inlet excitation) in these simulations. Indeed, we have verified that, as soon as this symmetry is broken, wavy rolls develop on the channel axis, as it is observed in the experiments [7].

Despite the just described differences, the frequency $f_{\approx}$, the axial wave length $\lambda \approx$ and the dimensionless phase velocity $c_{\approx}=\lambda_{\approx} f \approx / U_{\text {mean }}$ of the fully-established wavy rolls of Figures 8(a) 
and 8 (b) (see time $t=400$ ) are nearly equal. Indeed, whatever the inlet excitation amplitude is, the Fourier spectra of time signal $(T(t), U(t), V(t)$ or $W(t))$, at any point in the channel downstream, have a fundamental frequency at $3 \mathrm{~Hz}$ and harmonics of very small amplitude at $6 \mathrm{~Hz}$ and sometimes at $9 \mathrm{~Hz}$. Therefore, as expected (since the convectively unstable instabilities behave like noise amplifier [45]), the fundamental frequency $f_{\approx}$ of the wavy rolls is equal to the forcing frequency $f_{\text {pert }}=3 \mathrm{~Hz}$. Experimentally, Pabiou and Mergui $[7,41]$ obtain the same behavior: $f_{\approx}=f_{\text {pert }}=3 \mathrm{~Hz}$. The average axial wave length $\lambda \approx$ is numerically measured from the space profiles $T(x), U(x), V(x)$ or $W(x)$, at $(Y, Z)=(l / 2, H / 2)$, for $200 H \leq$ $X \leq 250 H$, when the inlet excitation amplitude is $v_{\text {max }}$ pert $=0.37$, and for $100 H \leq X \leq 250 H$, when $v_{\text {max-pert }}=3.7$ (see the space profiles of $W(x)$ shown on Figure 9 ). In the first case (for $v_{\text {max-pert }}=0.37$, we measure $\lambda_{\approx}=4.60 H \pm 0.04 H$ and thus $c_{\approx}=1.13 \pm 0.01$. In the second case (for $v_{\text {max }- \text { pert }}=3.7$ ), we measure $\lambda_{\approx}=4.68 H \pm 0.02 H$ and thus $c_{\approx}=1.15 \pm 0.005$. These values agree with the experiments since Pabiou and Mergui $(2003)[7,44]$ have measured a phase velocity $c_{\approx}=1.1 \pm 0.08$ and $\lambda_{\approx}=4.5 H \pm 0.3 H$.

\section{Code performance analysis}

Our code has been developed and optimized on vectorial computers. All the computations of the present paper were carried out on the NEC-SX5 vectorial super computer of CNRS (the French Scientific Research National Center): IDRIS, Orsay, France. This computer has got 8 vectorial registers of 256 words, that is to say that the maximum vectorization length is of 256 words of 64 bits, and its crest power (its maximum power when an addition and a multiplication are simultaneously done) is 8 Gflops ( $8 \times 10^{9}$ floating point operations per second). In this section, we analyze the global performance of this code, taking into account all the steps of the computation (initialization, preprocessing phase, solversolvinging and computing and writing of the output data) in order to show its efficiency.

For all the grids used and all the computations performed, the $G$ flops number varies between 5.4 and 6.2, when the average vectorization length varies between 233 and 254 words, and the unit CPU time per time step and per node, $t_{C P U-u n i t}$, varies between $1.45 \times 10^{-7} \mathrm{~s}$ and $1.85 \times 10^{-7} \mathrm{~s}$. Note that this time is almost independent of the time step and grid size since all the solving methods are direct methods. Thus, the total CPU time, $t_{C P U-t o t}$, to integrate

the discretized equations on a $N$ node grid, during $N_{t}$ time steps, is equal to: $t_{C P U-t o t}=$ 
$N N_{t} t_{C P U-u n i t}$. This time is given in Table 1 for the different test cases presented in subsection 4.2. To be more precise, for the simulations presented on Figure 8, carried out with a grid of 4.5 million nodes and with a dimensionless total integration time $t=400$, the code performances are: 6 Gflops, a vectorization length of 244 words, $t_{C P U-u n i t}=1.67 \times 10^{-7} \mathrm{~s}$ and $t_{C P U-t o t}=$ $4 h$ 10. Therefore, the results in Table 1 clearly show that the present code permits to solve unsteady 3D incompressible mixed convection flows, in high aspect ratio channels, for very reasonable time costs.

\section{Summary and conclusion}

In the present work, we have presented and validated an efficient vectorized finite difference code to solve the 3D incompressible Navier-Stokes equations, under the Boussinesq approximation, for mixed convection flows in high aspect ratio channels. Even though the methods used to solve these equations are relatively classic (Goda's algorithm, incremental factorization method of ADI type to solve the Helmholtz equations and diagonalization of the 1D Laplace operators to solve the Poisson equation), they are rarely used to compute incompressible mixed convection flows, as it is attested by literature on the subject. We have chosen such methods because they are all direct methods and because the resulting linear systems decompose into tridiagonal linear systems and can therefore be solved by the highly vectorizable TDMA algorithm.

The space and time convergence orders of the present code have been shown to be both of second order. A method consisting in imposing any analytical solution to the model-equations, by means of the source terms, and in comparing this analytical solution to the computed one has been developed to determine the space and time convergence orders.

The code is validated through comparisons with experiments on PRB flows. They concern steady longitudinal rolls, unsteady transverse rolls and, for the first time, wavy rolls. It is indeed the first time that wavy rolls, controlled by introducing sinusoidal excitations at channel inlet, are numerically obtained. All the experimental results are quantitatively reproduced by the numerical simulations when the experimental and numerical uncertainties are taken into account. It is particularly proved that the "strong" decrease, with the Rayleigh number, of the dimensionless phase velocity of the transversal rolls, observed in the experiments of Ouazzani et al. (1990) [5], is due to the lateral confinement ( $B=3.63$ in these experiments). However, it has been sometimes very difficult to finely reproduce the experimental results because the 
experimental operating conditions, particularly the thermal boundary conditions, are not well known. It is also difficult to well evaluate the experimental and numerical uncertainties on the measured quantities. This highlights the difficulties encountered when validating numerical codes with experimental results and the real lack of benchmark solutions for 3D incompressible mixed convection flows in channel. It is therefore useful and necessary that the community involved in numerical simulations of mixed convection flows fills this gap.

The whole code has been shown to be highly vectorizable and small time consuming since it is able to operate 6 Gflops on average (for a possible maximum of 8 Gflops), at a CPU time per node and per time step $t_{C P U-u n i t} \approx 1.6 \times 10^{-7} \mathrm{~s}$, on a NEC-SX5 vectorial super-computer. More precisely, the proposed code is able to compute unsteady mixed convection flows in very long channels, say with 200 or 300 streamwise aspect ratios, for about 4 hours total CPU time on grids of 4 million computational nodes. Thus, the numerical simulation of the space development of convectively unstable thermoconvective patterns, under the action of external excitations, will be able to be considered now. Note finally that, if the code presented in this paper has been specially tailored to study 3D PRB flows in long channels, it could easily be extended to other types of incompressible flows in channels or ducts too.

\section{Acknowledgements}

Drs E. Chénier and H. Pabiou are greatly acknowledged for numerous helpful discussions. All the computations were carried out on the NEC-SX5 super computer at IDRIS (Orsay, France) under research project $\mathrm{n}^{\circ} 41474$. The present work is supported by the French government through a Pluri-Formation Plan (PPF) on "Instabilities and transitions in thermoconvective flows in complex situations".

\section{References}

[1] R. M. Clever and F. H. Busse, Instabilities of longitudinal rolls in the presence of Poiseuille flow, J. Fluid Mech., vol. 229, pp. 517-529, 1991.

[2] H. W. Müller, M. Lücke, and M. Kamps, Transversal convection patterns in horizontal shear flow, Phys. Review A, vol. 45, pp. 3714-3725, 1992. 
[3] P. Carrière and P. A. Monkewitz, Convective versus absolute instability in mixed RayleighBénard-Poiseuille convection, J. Fluid Mech., vol. 384, pp. 243-262, 1999.

[4] K. C. Chiu and F. Rosenberger, Mixed convection between horizontal plates -1. Entrance effects, Int. J. Heat Mass Transfer, vol. 30, pp. 1645-1654, 1987.

[5] M. T. Ouazzani, J. K. Platten, and A. Mojtabi, Etude expérimentale de la convection mixte entre deux plans horizontaux à températures différentes -2, Int. J. Heat Mass Transfer, vol. 33 , pp. 1417-1427, 1990.

[6] C. H. Yu, M. Y. Chang, and T. F. Lin, Structures of moving transverse and mixed rolls in mixed convection of air in a horizontal plane channel, Int. J. Heat Mass Transfer, vol. 40, pp. 333-346, 1997.

[7] H. Pabiou, Mise en évidence expérimentale d'une instabilité convective dans un écoulement de Poiseuille-Rayleigh-Bénard. PhD thesis, The University of Paris VI - Pierre and Marie Curie, France, 2003.

[8] K. F. Jensen, E. O. Einset, and D. I. Fotiadis, Flow phenomena in chemical vapor deposition of thin films, Annu. Rev. Fluid Mech., vol. 23, pp. 197-233, 1991.

[9] X. Nicolas, Bibliographical review on the Poiseuille-Rayleigh-Bénard flows: the mixed convection flows in horizontal rectangular ducts heated from below (in French with abridged English version), Int. J. Thermal Sciences, vol. 41, pp. 961-1016, 2002.

[10] M. T. Ouazzani, J. K. Platten, and A. Mojtabi, Intermittent patterns in mixed convection, Applied Scientific Research, vol. 51, pp. 677-685, 1993.

[11] M. Y. Chang, C. H. Yu, and T. F. Lin, Flow visualization and numerical simulation of transverse and mixed vortex roll formation in mixed convection of air in a horizontal flat duct, Int. J. Heat Mass Transfer, vol. 40, pp. 1907-1922, 1997.

[12] E. Nobile and L. Onesti, Numerical simulation of time-dependent mixed convection in horizontal rectangular channels, Proceedings of the 2nd European Thermal Sciences Conference and 14th UIT National Heat Transfer Conference, Edizioni ETS, pp. 787-794, 1996.

[13] R. Spall, Unsteady mixed convection in horizontal ducts with applications to chemical vapor deposition processes, Int. Comm. Heat Mass Transfer, vol. 23, pp. 115-122, 1996. 
[14] C. H. Yu and T. F. Lin, Effects of aspect ratio on vortex flow patterns in mixed convection of air through a bottom-heated horizontal rectangular duct, Num. Heat Tranf. Part A, vol. 31 , pp. $745-764,1997$.

[15] H. Pabiou, X. Nicolas, S. Xin, and S. Mergui, Observations d'une instabilité convective apparaissant sous la forme de rouleaux sinueux dans un écoulement de Poiseuille-RayleighBénard, Mécanique et Industries, vol. 4, pp. 537-543, 2003.

[16] G. Evans and R. Greif, A study of traveling wave instabilities in a horizontal channel flow with applications to chemical vapor deposition, Int. J. Heat Mass Transfer, vol. 32, pp. 895-911, 1989.

[17] X. Nicolas, A. Mojtabi, and J. K. Platten, Two dimensional numerical analysis of the Poiseuille-Bénard flow in a rectangular channel heated from below, Phys. Fluids, vol. 9, pp. 337-348, 1997.

[18] G. Evans and R. Greif, Unsteady three-dimensional mixed convection in a heated horizontal channel with applications to chemical vapor deposition, Int. J. Heat Mass Transfer, vol. 34, pp. 2039-2051, 1991.

[19] E. Schröder and K. Bühler, Three-dimensional convection in rectangular domains with horizontal throughflow, Int. J. Heat Mass Transfer, vol. 38, pp. 1249-1259, 1995.

[20] S. S. Chen and A. S. Lavine, Laminar buoyancy induced flow structures in a bottom heated aspect ratio 2 duct with throughflow, Int. J. Heat Mass Transfer, vol. 39, pp. 1-11, 1996.

[21] R. L. Sani and P. M. Gresho, Résumé and remarks on the open boundary condition minisymposium, Int. J. Num. Methods Fluids, vol. 18, pp. 983-1008, 1994.

[22] A. Bottaro, Note on open boundary conditions for elliptic flows, Num. Heat Tranf. Part $B$, vol. 18 , pp. $243-256,1990$.

[23] M. H. Kobayashi, J. C. F. Pereira, and J. M. M. Sousa, Comparison of several open boundary numerical treatments for laminar recirculating flows, Int. J. Num. Methods Fluids, vol. 16, pp. 403-419, 1993. 
[24] X. Nicolas, P. Traore, A. Mojtabi, and J. P. Caltagirone, Augmented Lagrangian method and open boundary conditions in 2D simulation of Poiseuille-Bénard channel flow, Int. J. Num. Methods Fluids, vol. 25, pp. 265-283, 1997.

[25] I. Orlanski, A simple boundary condition for unbounded hyperbolic flows, J. Comput. Phys., vol. 21, pp. 251-269, 1976.

[26] H. K. Moffat and K. F. Jensen, Three-dimensional flow effects in silicon cvd in horizontal reactors, J. Electrochemical Society, vol. 135, pp. 459-470, 1988.

[27] J. Ouazzani and F. Rosenberger, Three-dimensional modelling of horizontal chemical vapor deposition 1. MOCVD at atmospheric pressure, J. Cristal Growth, vol. 100, pp. 545-576, 1990.

[28] C. R. Kleijn and C. J. Hoogendoorn, A study of 2-D and 3-D transport phenomena in horizontal chemical vapor deposition reactors, Chemical Engineering Science, vol. 46, pp. 321$334,1991$.

[29] C. Karki, P. S. Sathyamurthy, and S. V. Patankar, Three-dimensional mixed convection in a horizontal chemical vapor deposition reactor, J. Heat Transfer, vol. 115, pp. 803-806, 1993.

[30] U. Narusawa, Numerical analysis of mixed convection at the entrance region of a rectangular duct heated from below, Int. J. Heat Mass Transfer, vol. 36, pp. 2375-2384, 1993.

[31] I. Hosokawa, Y. Tanaka, and K. Yamamoto, Mixed convective flow with mass transfer in a horizontal rectangular duct heated from below simulated by the conditional fourier spectral analysis, Int. J. Heat Mass Transfer, vol. 36, pp. 3029-3042, 1993.

[32] C. H. Yu, M. Y. Chang, C. C. Huang, and T. F. Lin, Unsteady vortex roll structures in a mixed convective air flow through a horizontal plane channel: a numerical study, Int. J. Heat Mass Transfer, vol. 40, pp. 505-518, 1997.

[33] G. Evans and R. Greif, Thermally unstable convection with applications to chemical vapor deposition channel reactors, Int. J. Heat Mass Transfer, vol. 36, pp. 2769-2781, 1993.

[34] R. Spall, Observations of spanwise symmetry breaking for unsteady mixed convection in horizontal ducts, J. Heat Transfer, vol. 118, pp. 885-888, 1996. 
[35] Q. Wang, H. Yoo, and Y. Jaluria, Convection in a horizontal rectangular duct under constant and variable property formulations, Int. J. Heat Mass Transfer, vol. 46, pp. 297$310,2003$.

[36] S. Patankar, Numerical heat transfer and fluid flow. Hemisphere, Washington DC, 1980.

[37] K. Goda, A multistep technique with implicit difference schemes for calculating two- or three-dimensional cavity flows, J. Comput. Phys., vol. 30, pp. 76-95, 1979.

[38] C. Hirsch, Numerical computation of internal and external flows, vol. 1. chap. 11, Wiley Interscience, 1989.

[39] R. C. L. Bail, Use of fast fourier transforms for solving partial differential equations in physics, J. Comput. Phys., vol. 9, pp. 440-465, 1972.

[40] X. Nicolas, H. Pabiou, S. Xin, A. Benzaoui, S. Mergui, D. Gobin, and P. L. Quéré, Mise en évidence du caractère convectivement instable des rouleaux thermoconvectifs sinueux dans les écoulements de Poiseuille-Rayleigh-Bénard, Proceedings of the Thermal French Congress SFT2003, Grenoble, France, pp. 273-278, 2003.

[41] H. Pabiou and S. Mergui, Etude expérimentale d'une instabilité convective dans un écoulement de Poiseuille-Rayleigh-Bénard, Proceedings of the Thermal French Congress SFT2004, Presqu'̂̀le de Giens, France, vol. 1, pp. 277-282, 2004.

[42] X. Nicolas, J. M. Luijkx, and J. K. Platten, Linear stability of mixed convection flows in horizontal rectangular channels of finite transversal extension heated from below, Int. J. Heat Mass Transfer, vol. 43, pp. 589-610, 2000.

[43] M. Dubois and P. Bergé, Experimental study of the velocity field in Rayleigh-Bénard convection, J. Fluid Mech., vol. 85, pp. 641-653, 1978.

[44] H. Pabiou and S. Mergui, Personal communication, 2004.

[45] J. M. Chomaz, P. Huerre, and L. G. Redekopp, Bifurcations to local and global modes in spatially developing flows, Physical Review Letters, vol. 60, pp. 25-28, 1988. 


\begin{tabular}{|c|c|c|c|c|c|c|c|c|c|c|}
\hline test case & rolls & $A$ & $B$ & $N_{x}$ & $N_{y}$ & $N_{z}$ & $N$ & $\Delta t$ & $N_{t}$ & $t_{C P U-t o t}$ \\
\hline \hline $1(\S 4.2 .1)$ & $/ /$ & 40 & 9.65 & 480 & 120 & 32 & $1.85 \times 10^{6}$ & $5 \times 10^{-3}$ & 10000 & 45 min \\
\hline $2(\S 4.2 .2)$ & $/ /$ & 40 & 3.63 & 480 & 90 & 32 & $1.38 \times 10^{6}$ & $5 \times 10^{-3}$ & 6000 & 20 min \\
\hline $2(\S 4.2 .2)$ & $\perp$ & 27 & 3.63 & 400 & 60 & 30 & $0.72 \times 10^{6}$ & $2 \times 10^{-3}$ & 20000 & 40 min \\
\hline $3(\S 4.2 .3)$ & $\approx$ & 250 & 10.38 & 1250 & 120 & 30 & $4.5 \times 10^{6}$ & $2 \times 10^{-2}$ & 20000 & $4 h 10$ \\
\hline
\end{tabular}

Table 1: Parameters of the space and time discretizations for the simulations of the different test cases presented in subsection 4.2 and total CPU time $t_{C P U-t o t}$ necessary for their computing. 


\section{Figure captions}

Figure 1: Geometry and top and bottom thermal boundary conditions (the vertical lateral walls at $Y=0$ and $W$ are adiabatic).

Figure 2: Relative errors $\epsilon_{f}$ between the analytical and computed fields (a) as a function of $\Delta x$ for a steady flow and (b) as a function of $\Delta t$ for an unsteady flow. The space and time convergence orders are respectively given by the slopes of the curves $\log \left(\epsilon_{f}\right)=f(\log (\Delta x))$ and $\log \left(\epsilon_{f}\right)=f(\log (\Delta t))$. Two lines whose slope is equal to 2 are drawn for comparison.

Figure 3: Spanwise profiles of the axial velocity component $U$, at 3 different axial coordinates, at $Z=0.5 H$ (top) and at $Z=0.2 H$ (bottom), numerically computed by the present code (lines) and compared with the experimental results [4] (crosses) and with the numerical results [12] (dashed lines), in a longitudinal roll flow $(R e=42, \operatorname{Ra}=4878, \operatorname{Pr}=0.71)$.

Figure 4: Comparison of spanwise profiles of the vertical velocity component $W$, at $(x, z)=$ $(15.7,0.5)$, in a longitudinal roll flow at different Rayleigh numbers $(\operatorname{Re}=1.02, \operatorname{Pr}=6.4)$. Numerical results are in lines and the experimental results [5] are in symbols.

Figure 5: Instantaneous vertical velocity, $W$, vertical velocity envelop, $W_{e n}$, and average vertical velocity, $W_{a v}$, at $(Y, Z)=(l / 2, H / 2)$, as a function of $X(R a=4700, R e=0.21, \operatorname{Pr}=6.4)$.

Figure 6: Square of the saturation vertical velocity, $W_{s}^{2}$, as a function of $R a$, measured at $Y=l / 2$ and $Z=H / 2$ in a transversal roll flow, at $R e=0.21$ and $\operatorname{Pr}=6.4$. Comparison of the present numerical results with the experimental results of Ouazzani et al. (1990) [5].

Figure 7: Transversal roll phase velocity, $c_{\perp}$, as a function of the Rayleigh number $(\operatorname{Pr}=6.4$, $B=3.63$ ). Comparison of the experimental results of Ouazzani et al. (1990) [5], with the 2D numerical results of Nicolas et al. (1997) [17] and the present 3D numerical results.

Figure 8: Top view of computed temperature fields, $\theta$, in the plane $z=0.5$, at different times, during the development of wavy rolls, obtained by introducing a $3 \mathrm{~Hz}$ inlet sinusoidal excitation on the velocity profile for $t>0$ in a steady longitudinal roll flow $(\operatorname{Pr}=0.7, \operatorname{Ra}=6300$, $\left.R e=162, A=250, A_{e}=2, B=10.38\right)$. The darker lines correspond to both the hotter and colder temperatures and therefore to upward and downward flows: there is thus one roll between two dark lines. (a) Development of 10 wavy rolls for an excitation of amplitude $v_{\text {max-pert }}=0.37$. (b) Development of 12 wavy rolls for an excitation of amplitude $v_{\text {max }}$ pert $=3.7$.

Figure 9: Axial profiles of the vertical velocity $W(x)$, along the line $(Y, Z)=(l / 2, H / 2)$, for the two fully-established wavy roll flows presented on Figure 8 at time $t=400$. 


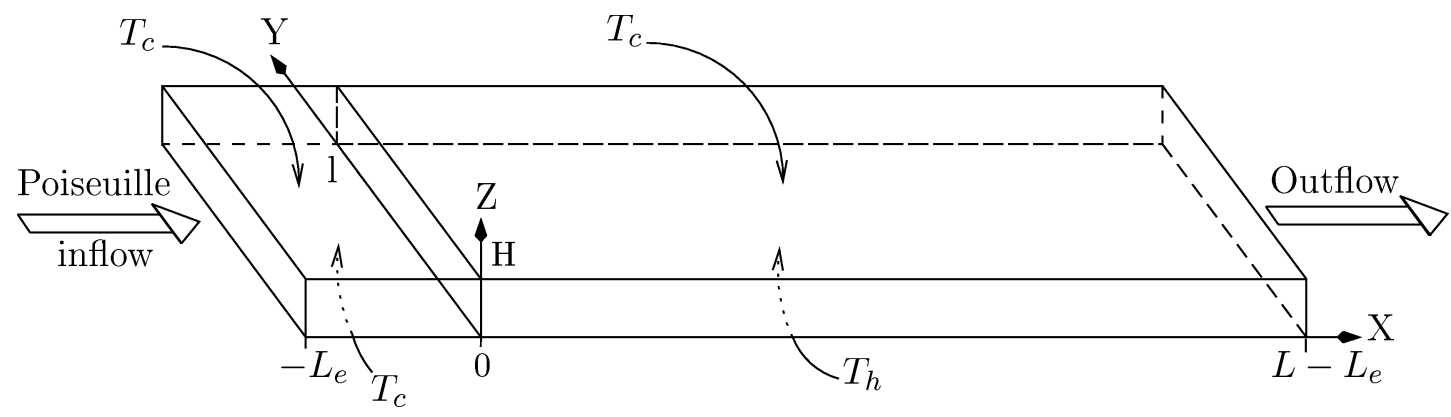

Figure 1: Geometry and top and bottom thermal boundary conditions (the vertical lateral walls at $Y=0$ and $W$ are adiabatic). 


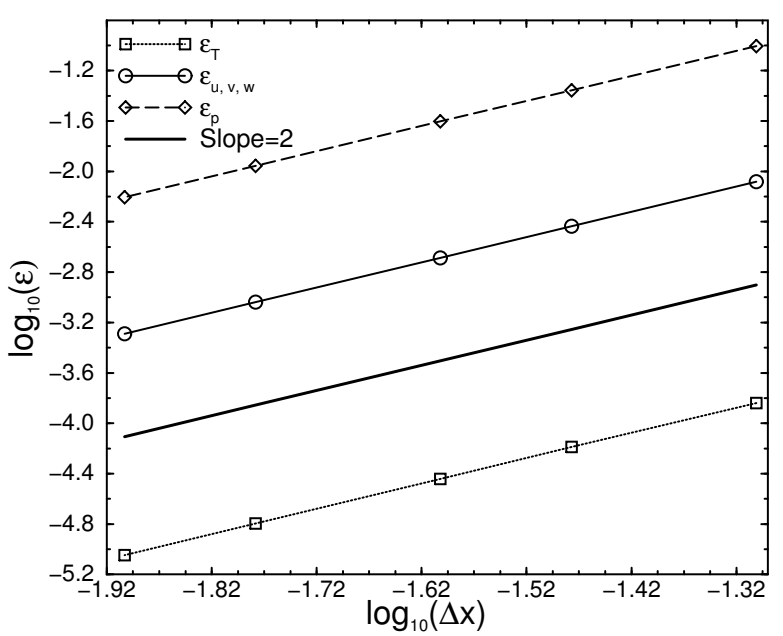

(a)

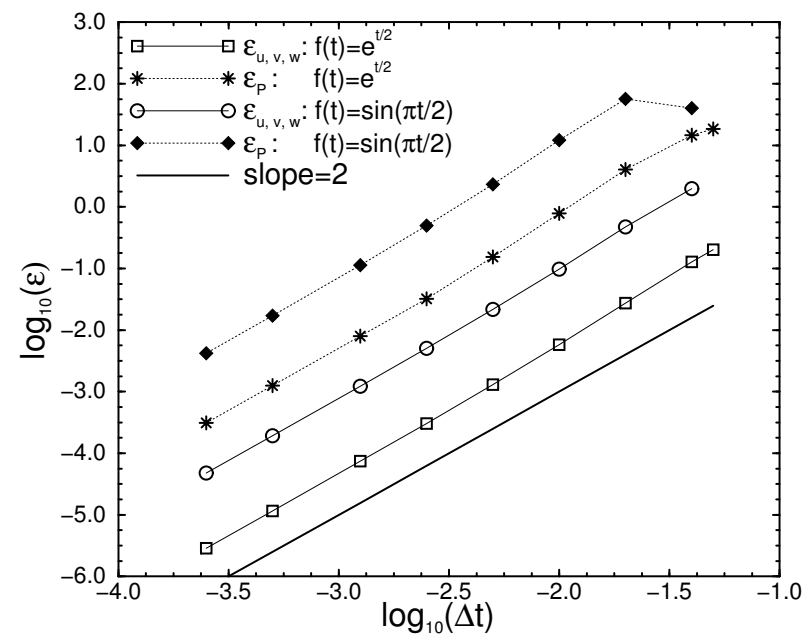

(b)

Figure 2: Relative errors $\epsilon_{f}$ between the analytical and computed fields (a) as a function of $\Delta x$ for a steady flow and (b) as a function of $\Delta t$ for an unsteady flow. The space and time convergence orders are respectively given by the slopes of the curves $\log \left(\epsilon_{f}\right)=f(\log (\Delta x))$ and $\log \left(\epsilon_{f}\right)=f(\log (\Delta t))$. Two lines whose slope is equal to 2 are drawn for comparison. 

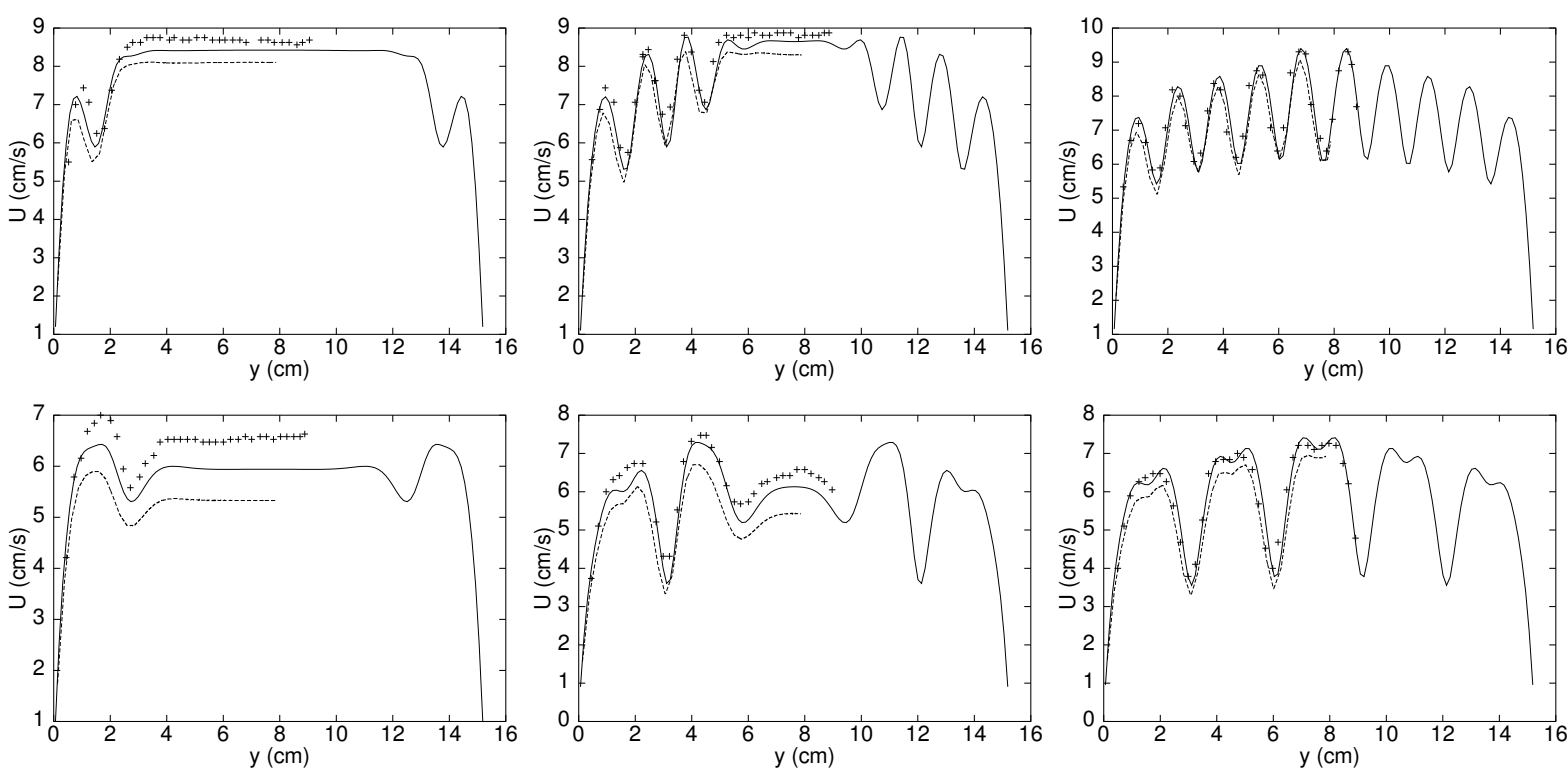

(a) $X=10 \mathrm{~mm}$

(b) $X=20 \mathrm{~mm}$

(c) $X=30 \mathrm{~mm}$

Figure 3: Spanwise profiles of the axial velocity component $U$, at 3 different axial coordinates, at $Z=0.5 H$ (top) and at $Z=0.2 H$ (bottom), numerically computed by the present code (lines) and compared with the experimental results [4] (crosses) and with the numerical results [12] (dashed lines), in a longitudinal roll flow ( $R e=42, R a=4878, \operatorname{Pr}=0.71$ ). 


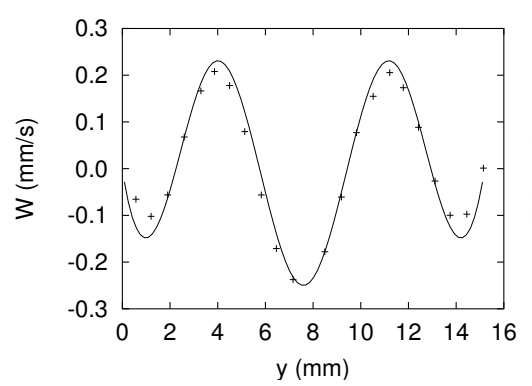

(a) $R a=2429$

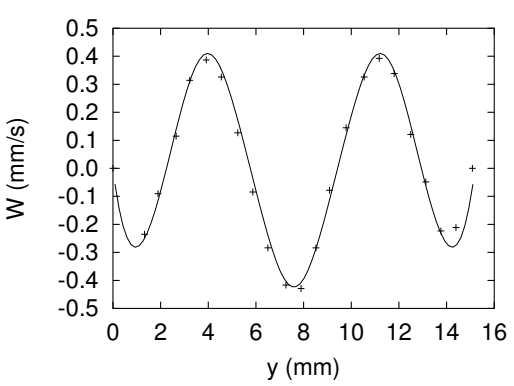

(b) $R a=3494$

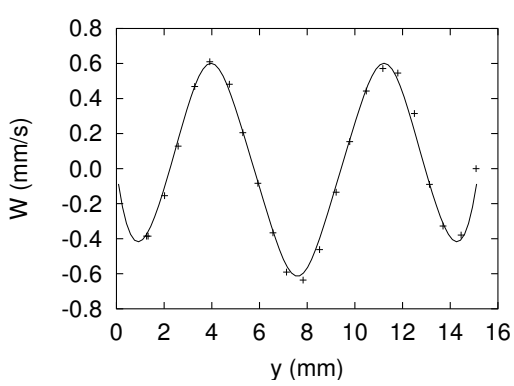

(c) $R a=4724$

Figure 4: Comparison of spanwise profiles of the vertical velocity component $W$, at $(x, z)=$ $(15.7,0.5)$, in a longitudinal roll flow at different Rayleigh numbers $(\operatorname{Re}=1.02, \operatorname{Pr}=6.4)$. Numerical results are in lines and the experimental results [5] are in symbols. 


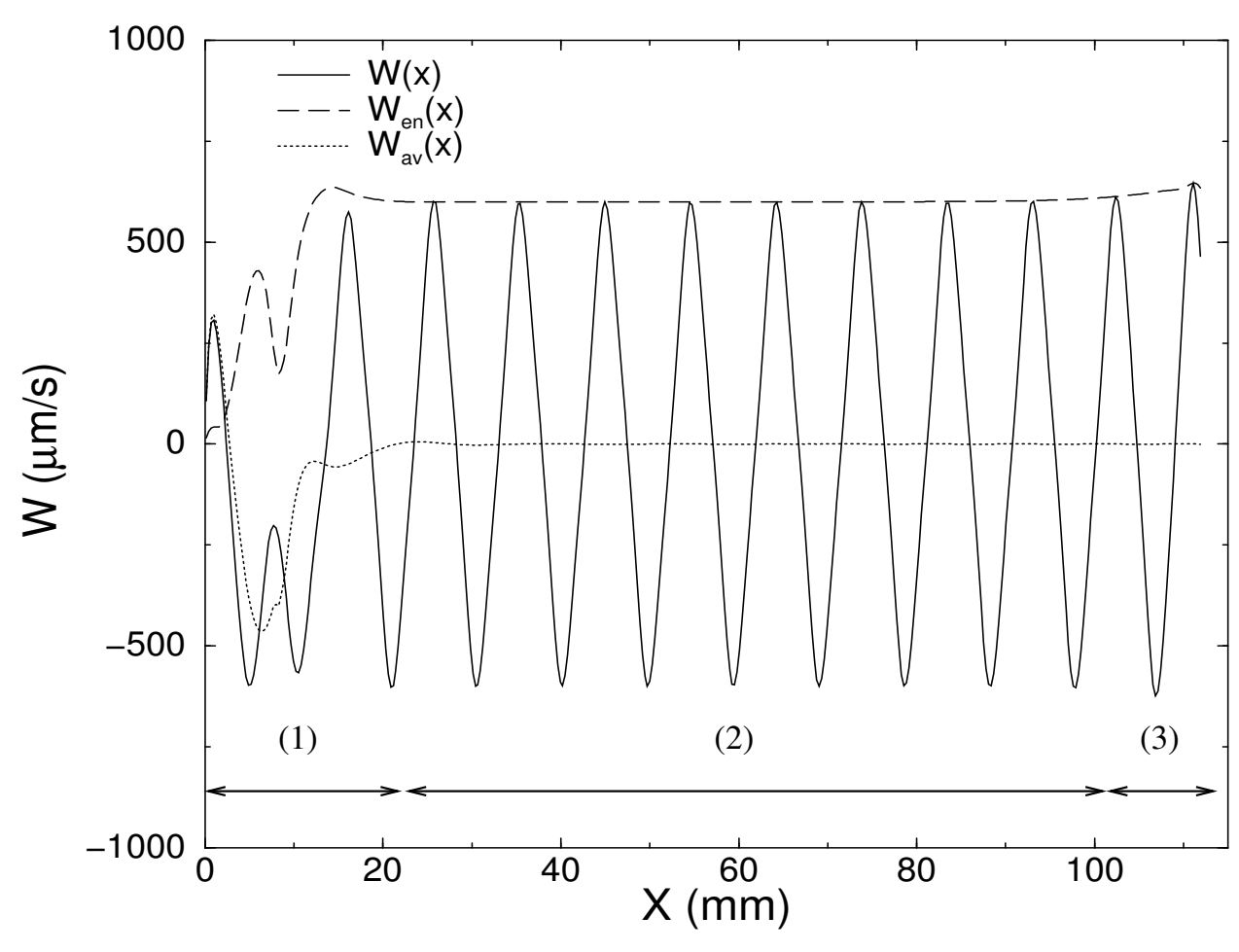

Figure 5: Instantaneous vertical velocity, $W$, vertical velocity envelop, $W_{e n}$, and average vertical velocity, $W_{a v}$, at $(Y, Z)=(l / 2, H / 2)$, as a function of $X(R a=4700, \operatorname{Re}=0.21, \operatorname{Pr}=6.4)$. 


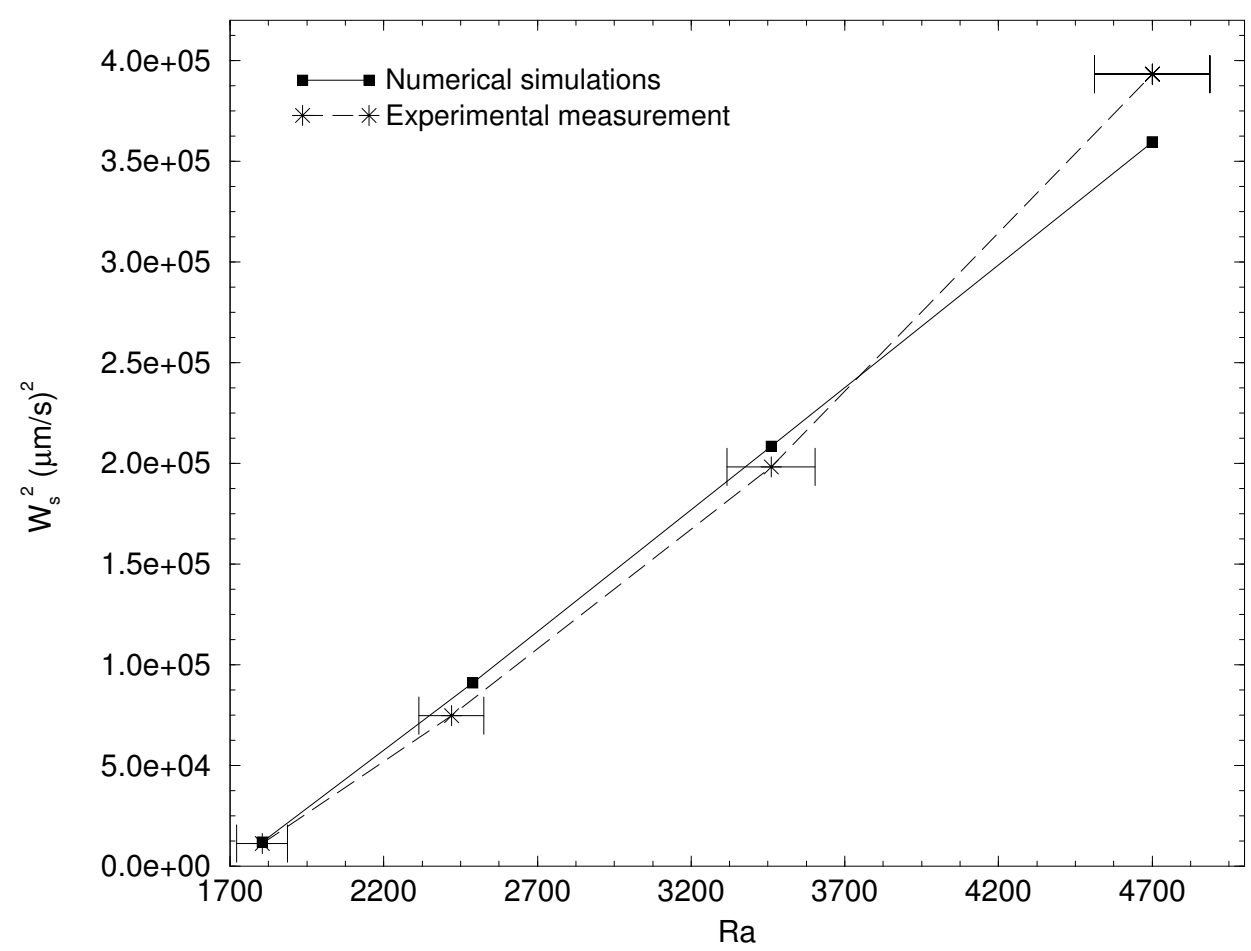

Figure 6: Square of the saturation vertical velocity, $W_{s}^{2}$, as a function of $R a$, measured at $Y=l / 2$ and $Z=H / 2$ in a transversal roll flow, at $\operatorname{Re}=0.21$ and $\operatorname{Pr}=6.4$. Comparison of the present numerical results with the experimental results of Ouazzani et al. (1990) [5]. 


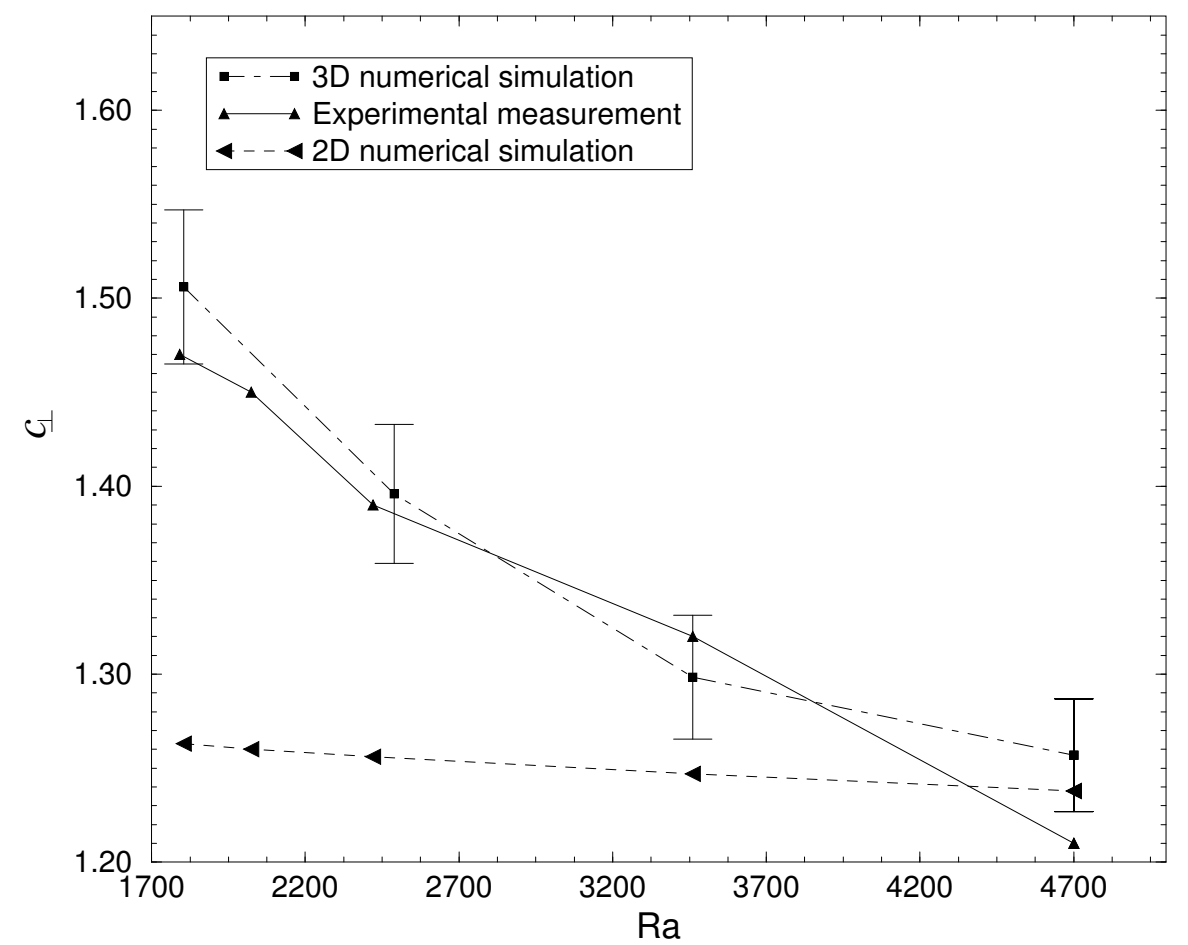

Figure 7: Transversal roll phase velocity, $c_{\perp}$, as a function of the Rayleigh number $(\operatorname{Pr}=6.4$, $B=3.63$ ). Comparison of the experimental results of Ouazzani et al. (1990) [5], with the 2D numerical results of Nicolas et al. (1997) [17] and the present 3D numerical results. 


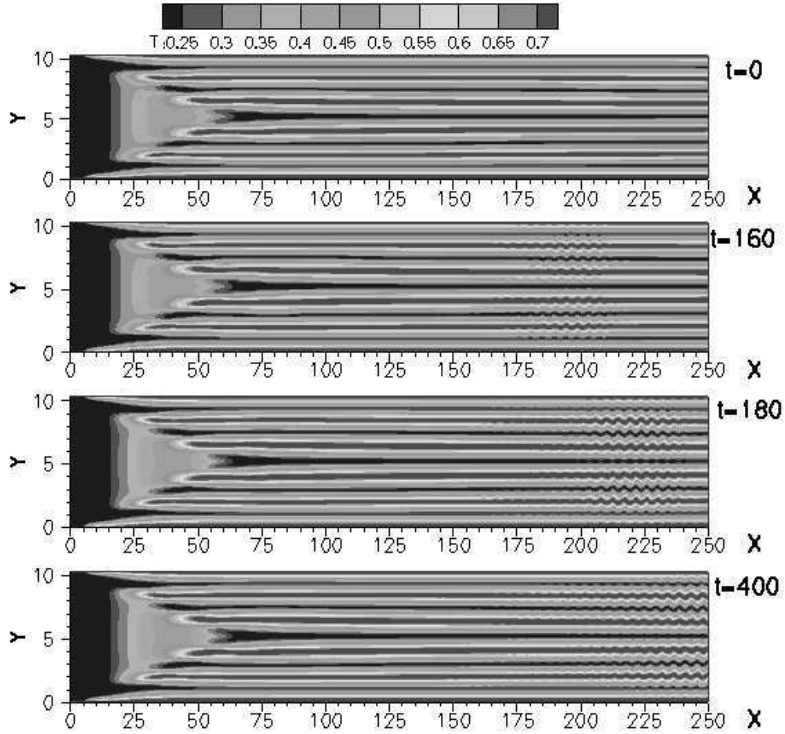

(a) $v_{\max -\text { pert }}=0.37$

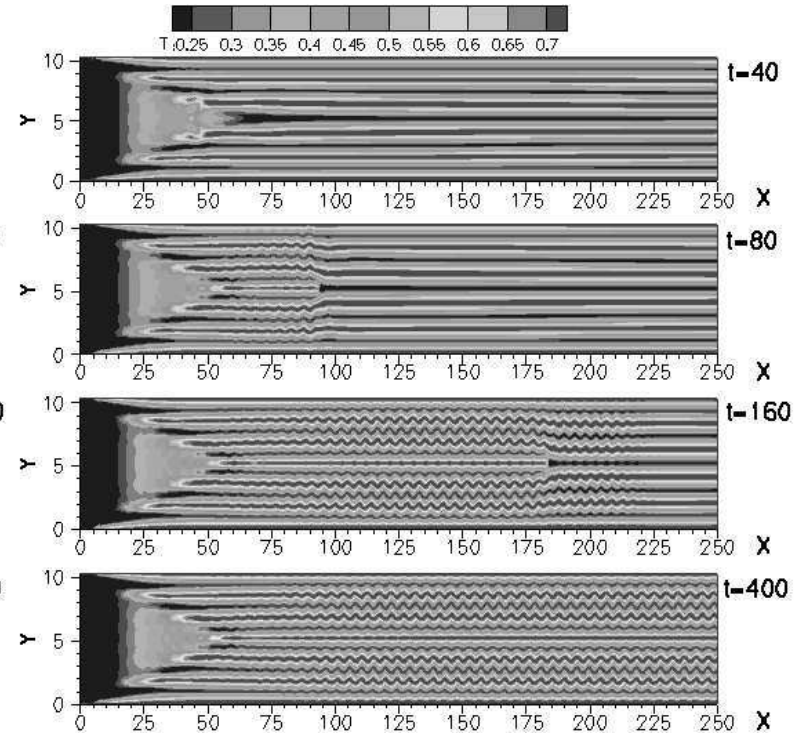

(b) $v_{\max -\text { pert }}=3.7$

Figure 8: Top view of computed temperature fields, $\theta$, in the plane $z=0.5$, at different times, during the development of wavy rolls, obtained by introducing a $3 \mathrm{~Hz}$ inlet sinusoidal excitation on the velocity profile for $t>0$ in a steady longitudinal roll flow $(\operatorname{Pr}=0.7, R a=6300, R e=162$, $\left.A=250, A_{e}=2, B=10.38\right)$. The darker lines correspond to both the hotter and colder temperatures and therefore to upward and downward flows: there is thus one roll between two dark lines. (a) Development of 10 wavy rolls for an excitation of amplitude $v_{\text {max-pert }}=0.37$. (b) Development of 12 wavy rolls for an excitation of amplitude $v_{\text {max }}$ pert $=3.7$. 


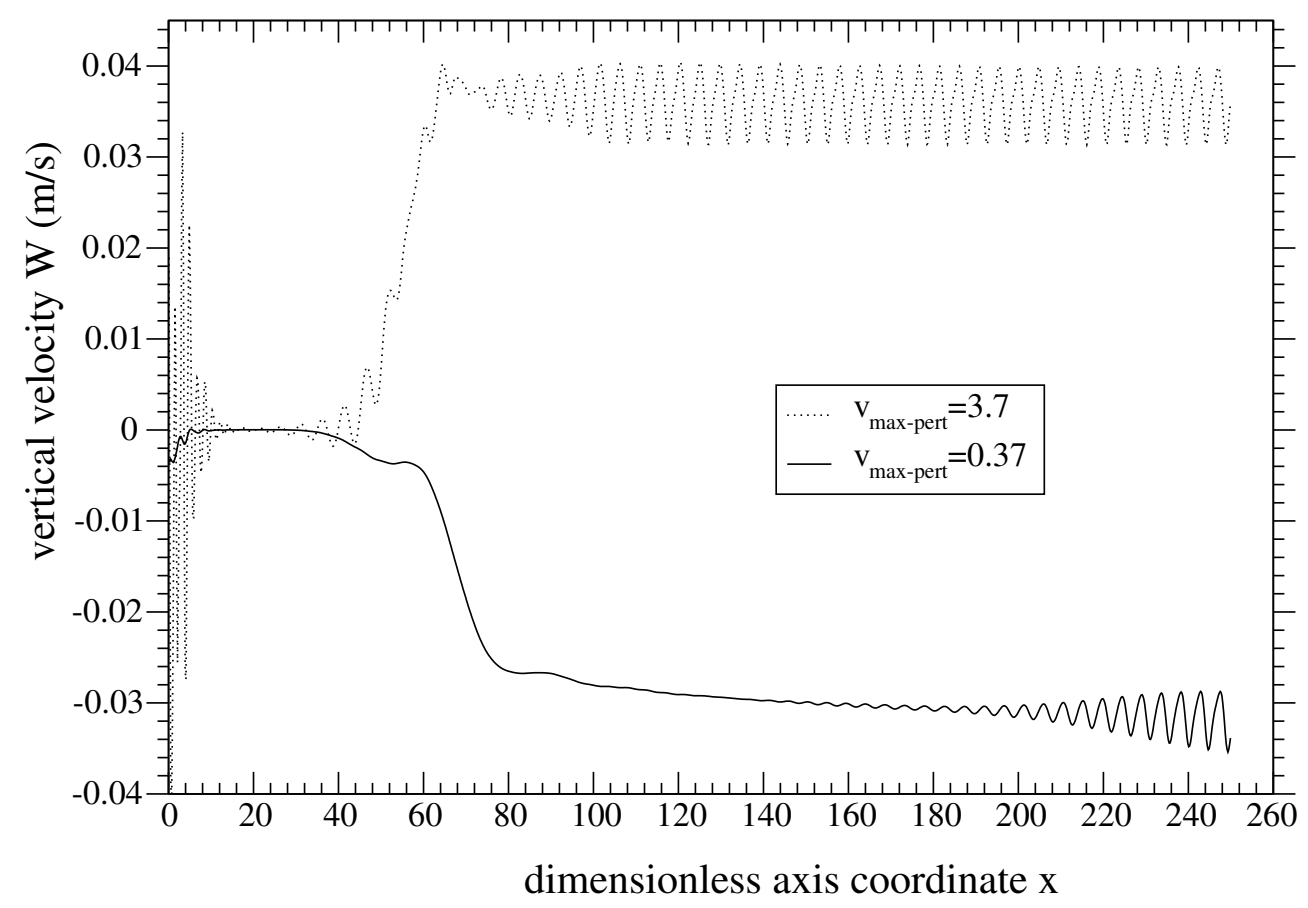

Figure 9: Axial profiles of the vertical velocity $W(x)$, along the line $(Y, Z)=(l / 2, H / 2)$, for the two fully-established wavy roll flows presented on Figure 8 at time $t=400$. 